\title{
Probing the two light Higgs scenario in the NMSSM with a low-mass pseudoscalar
}

\author{
David G. Cerdeño, ${ }^{a, b}$ Pradipta Ghosh ${ }^{a, b}$ and Chan Beom Park ${ }^{c}$ \\ ${ }^{a}$ Departamento de Fúsica Teórica, Universidad Autónoma de Madrid, \\ Cantoblanco, E-28049 Madrid, Spain \\ ${ }^{b}$ Instituto de Física Teórica UAM/CSIC, Universidad Autónoma de Madrid, \\ Cantoblanco, E-28049 Madrid, Spain \\ ${ }^{c}$ TH Division, Physics Department, CERN, \\ CH-1211 Geneva 23, Switzerland \\ E-mail: davidg.cerdeno@uam.es, pradipta.ghosh@uam.es, \\ chanbeom.park@cern.ch
}

ABSTRACT: In this article we propose a simultaneous collider search strategy for a pair of scalar bosons in the NMSSM through the decays of a very light pseudoscalar. The massive scalar has a mass around $126 \mathrm{GeV}$ while the lighter one can have a mass in the vicinity of $98 \mathrm{GeV}$ (thus explaining an apparent LEP excess) or be much lighter. The successive decay of this scalar pair into two light pseudoscalars, followed by leptonic pseudoscalar decays, produces clean multi-lepton final states with small or no missing energy. Furthermore, this analysis offers an alternate leptonic probe for the $126 \mathrm{GeV}$ scalar that can be comparable with the $Z Z^{*}$ search channel. We emphasize that a dedicated experimental search for multi-lepton final states can be an useful probe for this scenario and, in general, for the NMSSM Higgs sector. We illustrate our analysis with two representative benchmark points and show how the LHC configuration with $8 \mathrm{TeV}$ center-of-mass energy and $25 \mathrm{fb}^{-1}$ of integrated luminosity can start testing this scenario, providing a good determination of the light pseudoscalar mass and a relatively good estimation of the lightest scalar mass.

KeYWORDS: Supersymmetry Phenomenology

ARXIV EPRINT: 1301.1325 


\section{Contents}

1 Introduction 1

2 Higgs signals with a low-mass pseudoscalar in the NMSSM 3

$\begin{array}{lll}3 & \text { Experimental constraints and the choice of parameters } & 6\end{array}$

$\begin{array}{llr}4 & \text { Collider analysis } & 9\end{array}$

$\begin{array}{llr}5 & \text { Conclusions } & 14\end{array}$

\section{Introduction}

The experimental evidence for a scalar boson with a mass around $126 \mathrm{GeV}[1,2]$ has been received with enthusiasm by the particle physics community, since it could correspond to the missing piece of the standard model (SM), the Higgs boson. Although most of the properties of this new particle (in particular the branching ratios of various decay modes) are compatible with a SM-like Higgs, an apparent di-photon excess [3-6], has been considered as a possible hint for physics beyond the SM, such as supersymmetry (SUSY). However, this excess remains yet to be confirmed [7].

It has been pointed out that in order to reproduce the mass of the Higgs boson in the Minimal supersymmetric Standard Model (MSSM) one generally needs a very heavy spectrum, raising concerns about the naturalness of this construction. This problem can be alleviated in extended supersymmetric constructions. In particular, the Next-to-Minimal supersymmetric Standard Model (NMSSM) can accommodate a $126 \mathrm{GeV}$ scalar [8-25], while maintaining the idea of naturalness [25-31]. The advantage of the NMSSM resides in the presence of an extra singlet field, which provides an additional contribution to the tree-level Higgs mass [32-36]. Thus, contrary to what happens in the MSSM, loop corrections can be smaller and the correct Higgs mass is achieved for a lighter supersymmetric spectrum. This extra singlet superfield $\hat{S}$ is introduced to promote the bilinear $\mu$-term $\mu \hat{H}_{d} \hat{H}_{u}$ in the MSSM to a trilinear coupling $\lambda \hat{S} \hat{H}_{d} \hat{H}_{u}$. After electroweak symmetry breaking (EWSB) takes place, the vacuum expectation value (VEV) of the singlet field triggers an effective $\mu$ parameter, $\lambda v_{s}$, which is naturally of the order of the electroweak scale, thus curing the $\mu$-problem of the MSSM [37]. On top of this, the singlet-doublet mixing of the resulting scalar states is useful to produce the observed di-photon excess [8-10, 38, 39].

The new singlet superfield induces one extra CP-even and CP-odd neutral scalars as well as one additional neutral fermion, thereby leading to a very rich phenomenology. In particular, a light pseudoscalar Higgs is viable if its singlet component is large [40-48]. Similarly, the lightest supersymmetric particle (LSP) can also be singlino-like [49-55] or, 
in general, a mixed state with interesting implications for dark matter searches (see, e.g., refs. $[56,57])$.

The presence of light scalar and pseudoscalar Higges can lead to characteristic Higgsto-Higgs cascade decays [40, 41]. Although these cascades are generally suppressed, a substantial enhancement can occur when particles are light and therefore induce distinctive signatures. ${ }^{1}$ For example, if the lightest pseudoscalar, denoted as $a_{1}^{0}$, has a mass $m_{a_{1}^{0}} \gtrsim 2 m_{b}$, the process $h_{1}^{0} \rightarrow 2 a_{1}^{0} \rightarrow 2 b \bar{b}$ with $4 b$ final states is dominant [42, 43, 66-70]. This seems a good channel for collider study, ${ }^{2}$ however, in hadron colliders like the LHC this signature is hidden by a huge QCD background. A viable alternative is looking for $4 \tau[40,42,43,46,58,65,69,71,72]$ or $2 \mu 2 \tau$ [72] final states, for which the problem of large QCD backgrounds is somehow ameliorated. However, this approach suffers from the poor tau-identification efficiency, dependent on the transverse momentum [73]. Besides, since tau decays are always accompanied by neutrinos, there is no sharp peak of $a_{1}^{0}$ invariant mass. For these reasons, as discussed in the literature, $4 \mu[69,74,75], 4 \gamma[41,63]$ or $2 \gamma+2$-jets [76] search channels with $m_{a_{1}^{0}}<2 m_{\tau}$ turn out to be the ideal way to probe NMSSM Higgses with very light pseudoscalars.

Recently, the possibility of having two light scalar Higgses in NMSSM has received a lot of attention. One of these Higgses, $h_{2}^{0}$, would correspond to the scalar observed by ATLAS and CMS with a mass around $126 \mathrm{GeV}$ and the other one, $h_{1}^{0}$, with a mass around $98 \mathrm{GeV}$ [24], would be consistent with the small excess in the LEP search for $e^{+} e^{-} \rightarrow Z h$, $h \rightarrow b \bar{b}[77-79] .^{3}$ Alternative scenarios with an even lighter $h_{1}^{0}$ have also been considered earlier $[42,43,83]$. Since the recent LHC observation of the $126 \mathrm{GeV}$ scalar, which is well compatible with a SM-like Higgs boson, many of these studies have become extremely constrained, although a small window for new physics is still open if the di-photon excess is confirmed. On the other hand, scenarios with very light pseudoscalars are also affected by stringent experimental bounds. This is the case for $m_{a_{1}^{0}}<2 m_{b}$ (which leads to leptonic final states $4 \mu, 2 \mu 2 \tau$, and $4 \tau)[47,64,72,84]$, with tight constraints in the case $m_{a_{1}^{0}}<2 m_{\tau}$ for CP-even Higgs masses in the range of $86-150 \mathrm{GeV}$ by the recent CMS analysis [85], especially in the case of singlet-like states. The latter is a consequence of the fact that $\operatorname{BR}\left(h_{1}^{0} / h_{2}^{0} \rightarrow a_{1}^{0} a_{1}^{0}\right) \sim 1$ and thus $\sigma\left(p p \rightarrow h_{1}^{0} / h_{2}^{0} \rightarrow 2 a_{1}^{0} \rightarrow 4 \mu\right)$ is sizable and would have already been observed. ${ }^{4}$

In this work we investigate potential detection channels for scenarios involving very light pseudoscalar particles and two light scalar Higgses in the NMSSM. For concreteness, we consider a SM-like scalar Higgs in the range $124 \mathrm{GeV}<m_{h_{2}^{0}}<128 \mathrm{GeV}$ (consistent with LHC findings) and a lighter $h_{1}^{0}$ in the mass range $96-100 \mathrm{GeV}$ (to account for the LEP excess) or lighter than $86 \mathrm{GeV}$ (to avoid the current CMS limit). In order to avoid the above mentioned constraints that affect the lightest pseudoscalar, $a_{1}^{0}$ is assumed to have

\footnotetext{
${ }^{1}$ To some extent, a scenario like this is a good illustration of the "no-lose" theorem for the NMSSM [40$45,58-65]$.

${ }^{2}$ Another possible search channel is $2 b 2 \tau$.

${ }^{3} \mathrm{~A}$ two light Higgs but without a low-mass pseudoscalar has been discussed in the MSSM [80, 81], as well as in the NMSSM [82].

${ }^{4}$ One way to avoid this is to consider the case when the production cross section for a singlet-like $h_{1}^{0} / h_{2}^{0}$ is very suppressed.
} 
a mass in the range $2 m_{\tau} \lesssim m_{a_{1}^{0}}<2 m_{b}$, since the $\mu^{+} \mu^{-}$final state is then a sub-leading leptonic mode with a branching ratio of the order of $10^{-2}$ and $\sigma\left(p p \rightarrow h_{1}^{0} \rightarrow 2 a_{1}^{0} \rightarrow 4 \mu\right)$ is small enough to elude the CMS limit of the $4 \mu$ search. We point out that the SM-like Higgs decay modes $h_{2}^{0} \rightarrow 2 a_{1}^{0} \rightarrow 4 \mu, 2 \mu 2 \tau$, and $4 \tau$ have branching fractions comparable to those for the same final states from $h_{2}^{0} \rightarrow Z Z^{*}$ and therefore provide alternative four-lepton signatures accompanied with zero or a small missing energy at the LHC. We also note that the long Higgs-to-Higgs cascades of $h_{2}^{0} \rightarrow h_{1}^{0} h_{1}^{0} \rightarrow 4 a_{1}^{0}$ can be open if $m_{h_{1}^{0}}<m_{h_{2}^{0}} / 2$, which constitutes a clean and distinctive multi-lepton signal at the LHC. We design a set of cuts that isolates the signal events from the background, study the distributions of the kinematic variable $M_{\mathrm{T} 2}[86,87]$, and determine to what extent the masses of the pseudoscalar and scalar Higgs particles can be reconstructed. In our analysis we include constraints from $B$-physics [88-93], the muon anomalous magnetic moment [94-98], and the LHC result for $h_{2}^{0} \rightarrow \gamma \gamma$. In addition, we impose the WMAP bound on the lightest neutralino relic abundance [99] and we ensure that its spin-dependent scattering cross section is consistent with XENON100 data [100].

The paper is organized as follows. We start with a brief description of the model and determine the relevant signatures in section 2. The choice of model parameters is justified in section 3 where we also describe the details of the analysis. Section 4 is dedicated to the analysis of the numerical results. We also introduce some discriminating variables to suppress SM backgrounds and we investigate the statistical significance of the proposed signals. Finally we summarize our conclusions in section 5 .

\section{Higgs signals with a low-mass pseudoscalar in the NMSSM}

The superpotential of the $\mathbb{Z}_{3}$-symmetric NMSSM is given by

$$
W=W^{\prime}-\epsilon_{a b} \lambda \hat{S} \hat{H}_{d}^{a} \hat{H}_{u}^{b}+\frac{1}{3} \kappa \hat{S} \hat{S} \hat{S}
$$

where $W^{\prime}$ is the MSSM superpotential without the $\mu$-term, $\hat{S}$ is a superfield singlet under the SM gauge group, and $a, b=1,2$ are $\mathrm{SU}(2)_{L}$ indices. Similarly, with $\mathcal{L}_{\text {soft }}^{\prime}$ representing the MSSM soft-terms without the $B_{\mu}$-term, the Lagrangian containing the soft SUSYbreaking terms in a supergravity framework is given by

$$
-\mathcal{L}_{\text {soft }}=-\mathcal{L}_{\text {soft }}^{\prime}+m_{S}^{2} \widetilde{S}^{*} \widetilde{S}-\epsilon_{a b}\left(\lambda A_{\lambda}\right) \widetilde{S} H_{d}^{a} H_{u}^{b}+\frac{1}{3}\left(\kappa A_{\kappa}\right) \widetilde{S} \widetilde{S} \widetilde{S}+\text { H.c. }
$$

The last trilinear term in eq. (2.1) is essential to avoid an unacceptable axion associated with the breaking of a global U(1) symmetry [33]. After EWSB, this term also generates an effective Majorana mass $2 \kappa v_{s}$ for the singlino-like neutralino. The second term of eq. (2.1) not only generates an effective $\mu$ parameter, $\mu_{\text {eff }}=\lambda v_{s}$, but also provides an extra treelevel contribution to the lightest doublet-like Higgs boson mass. The complete expression is now $[32-36]$,

$$
m_{h^{0}}^{2} \leq M_{Z}^{2} \cos ^{2} 2 \beta+3.62 M_{Z}^{2} \lambda^{2} \sin ^{2} 2 \beta
$$


where $\tan \beta=v_{u} / v_{d}$ is the ratio of up and down-type Higgs VEVs. Thus even maintaining perturbativity $(\lambda \lesssim 0.7)$, the extra contribution to the tree-level Higgs mass can be sizable for small values of $\tan \beta$. The last term of eq. (2.2) is important to understand the phenomenology of singlet-like scalars. In particular, when the lightest CP-even scalar $h_{1}^{0}$ as well as the lightest CP-odd scalar $a_{1}^{0}$ are predominantly singlet-like, the decay width for $h_{1}^{0} \rightarrow a_{1}^{0} a_{1}^{0}$ is proportional to $A_{\kappa} \kappa$.

As already mentioned in the previous section, the Higgs sector of the NMSSM is richer than that of the MSSM, featuring three CP-even scalar states, $\left(h_{1}^{0}, h_{2}^{0}, h_{3}^{0}\right)$ and two pseudoscalars, $\left(a_{1}^{0}, a_{2}^{0}\right)$. This is of particular interest for studying Higgs cascade decays, which can now be long and often result in multi-particle final states with no missing energy. The decay chains become more interesting in the presence of light scalar and pseudoscalar Higgses, which can be in agreement with present constraints if their singlet composition is dominant. In particular, a viable hierarchy for the scalar mass eigenstates in the NMSSM consists of having a light singlet-like $h_{1}^{0}$, a SM-like Higgs $h_{2}^{0}$ with a mass around $126 \mathrm{GeV}$ and a much heavier $h_{3}^{0}$ which is also doublet-like. This structure with two light scalar Higgses might produce distinctive features in collider searches, as compared to the signals that come from the MSSM Higgs sector, and therefore we concentrate on this possibility.

Although very light singlet-like pseudoscalars can give rise to potentially characteristic signatures, one must be careful with recent experimental constraints. In particular, for a scalar Higgs mass in the mass range of $86-150 \mathrm{GeV}$, the upper limit of $\sigma\left(p p \rightarrow h_{1}^{0} / h_{2}^{0} \rightarrow\right.$ $\left.2 a_{1}^{0}\right) \times \mathrm{BR}^{2}\left(a_{1}^{0} \rightarrow \mu^{+} \mu^{-}\right)$has been found to be $3-5 \mathrm{fb}$, depending on the pseudoscalar mass for $1 \mathrm{GeV}<m_{a_{1}^{0}}<2 m_{\tau}$ in the $7 \mathrm{TeV}$ CMS search [85]. Thus, if we want to study the 98 $+126 \mathrm{GeV}$ scenario for the scalar Higgs bosons, in order to avoid this constraint, we will consider a light pseudoscalar with a mass in the range $2 m_{\tau} \lesssim m_{a_{1}^{0}}<2 m_{b}$. We call this Scenario I.

Another way to avoid the CMS bound is to consider a lighter scalar Higgs with $m_{h_{1}^{0}}<$ $86 \mathrm{GeV}$. Although this would relax the LHC limit on the pseudoscalar mass, in fact $2 m_{\tau}<$ $m_{a_{1}^{0}}<2 m_{b}$ is still very constrained from the LEP limit on $4 \tau$ [101] and multi-lepton/jet final states $[66,102,103]$ in the case when $m_{h_{1}^{0}} \lesssim m_{h_{2}^{0}} / 2$. We have included these constraints in our analysis and checked that viable points can still be obtained within this mass range for the lightest pseudoscalar. We call this Scenario II.

For clearness we summarise here the properties of the two kind of scenarios considered in our work

(I) $124 \mathrm{GeV} \lesssim m_{h_{2}^{0}} \lesssim 128 \mathrm{GeV}$, $96 \mathrm{GeV} \lesssim m_{h_{1}^{0}} \lesssim 100 \mathrm{GeV}$, $2 m_{\tau} \lesssim m_{a_{1}^{0}}<2 m_{b}$

(II) $124 \mathrm{GeV} \lesssim m_{h_{2}^{0}} \lesssim 128 \mathrm{GeV}$, $m_{h_{1}^{0}} \lesssim m_{h_{2}^{0}} / 2$

$2 m_{\tau} \lesssim m_{a_{1}^{0}}<2 m_{b}$

We have also checked that these benchmark scenarios are in agreement with ATLAS and CMS direct pseudoscalar searches, $p p \rightarrow a_{1}^{0} \rightarrow \mu^{+} \mu^{-}[104,105]$, due to the singlet nature of $a_{1}^{0}$ and its dominant decay branching fraction to $\tau$. 
We are now ready to specify the potential signatures that we will study for these scenarios. We consider the following decay modes,

$$
\begin{aligned}
& h_{1}^{0} \rightarrow a_{1}^{0} a_{1}^{0} \rightarrow 4 \ell+\mathscr{E}_{\mathrm{T}}, \\
& h_{2}^{0} \rightarrow a_{1}^{0} a_{1}^{0} \rightarrow 4 \ell+\mathbb{E}_{\mathrm{T}},
\end{aligned}
$$

where $\ell=e, \mu, \tau$. We here assume that $h_{1}^{0}$ and $h_{2}^{0}$ have been produced through gluon-fusion and consider inclusive decay modes of the tau lepton. The missing energy $\mathbb{E}_{\mathrm{T}}$ is associated with neutrinos coming from tau-decays and is generally small. It is important to emphasize that direct-pseudoscalar decays to a muon-pair will eventually yield a clean four-muon final state with vanishing missing energy and the only source of electrons is leptonic $\tau$-decay, since $\operatorname{BR}\left(a_{1}^{0} \rightarrow e^{+} e^{-}\right) \sim 0$. The second decay mode can act as an alternative source of fourlepton final state apart from $h_{2}^{0} \rightarrow Z Z^{*} \rightarrow 4 \ell$. In the chosen corner of parameter space, we found that the predicted $\operatorname{BR}\left(h_{2}^{0} \rightarrow a_{1}^{0} a_{1}^{0}\right)$ is comparable with that of $\operatorname{BR}\left(h_{2}^{0} \rightarrow Z Z^{*}\right)$, which still matches well the SM prediction. We thus encourage our experimental colleagues to search for possible excesses in $4 \mu, 4 \tau$ or $2 \mu 2 \tau$ channels by relaxing the $Z$-boson resonance condition. In the remainder of the paper we carry out a dedicated analysis including the discussion of SM backgrounds and useful collider variables to search for the four-lepton collider signals of these decay modes.

Concerning possible SUSY backgrounds, these generally lead to large missing energy and can therefore be distinguished with a cut on $\mathbb{E}_{\mathrm{T}}$. There can also be sources of fourlepton final states with a small or no missing energy, provided that one considers $R$-parity violation [106-112] in the NMSSM [113-119] or $\mu \nu \operatorname{SSM}$ [120-122]. It is well known that $R$-parity conserving SUSY models are characterized by large missing energy due to the production of a heavy neutralino LSP, which is stable and escapes the detector, whereas this is not the case in $R$-parity violating scenarios. Models with broken $R$-parity and non-minimal superfield content, on the other hand, exhibit moderate or large displaced vertices with non-prompt jets or leptons in the final states (see, for example, refs. [123-126]) with more complex Higgs cascade decays [126] and occasional correlations with neutrino physics [122-124] following [127-132], which can be used for discrimination. We also note that four leptons can be produced in $h_{3}^{0}$ decays, but with a very reduced production cross section.

Notice that one distinguishing feature of Scenario II is a three-step cascade decay of the Higgs boson $h_{2}^{0} \rightarrow 2 h_{1}^{0} \rightarrow 4 a_{1}^{0} \rightarrow$ multi-lepton/jet. We have checked for several points in the parameter space that, even when $m_{h_{1}^{0}} \gtrsim m_{h_{2}^{0}} / 2$, the branching ratio of $h_{2}^{0} \rightarrow h_{1}^{0} h_{1}^{0 *} \rightarrow$ $4 a_{1}^{0}$ can be as large as $\sim 10^{-3}$ and thus multi-lepton final states can be experimentally accessible. Due to the large number of leptons and/or jets in the final state, the main backgrounds are expected to be SUSY processes (such as cascade decays mediated by neutralinos and/or charginos), rather than SM ones. The analysis of this decay signal will be carried out in a separate work [133] since the search strategy is very different than for the other two. 


\section{Experimental constraints and the choice of parameters}

The recent discovery of a $126 \mathrm{GeV}$ scalar along with the reported di-photon excess set stringent limitations on the NMSSM parameter space. In addition, considering bounds from low-energy observables in flavour physics, the supersymmetric contribution to the muon anomalous magnetic moment $a_{\mu}^{\mathrm{SUSY}}$, and the relic abundance of the dark matter, which in our case would correspond to the lightest neutralino, narrow down significantly the viable regions.

In our analysis we impose compatibility with all these constraints. The numerical results are obtained with NMSSMTOOLS 3.2.1 [134-136], which calculates the mass spectrum and provides predictions for low-energy observables, as well as computing the decay widths of Higgses [137-140] and sparticles [141-143]. The masses of Higgs bosons include full two-loop contributions. We also consider the lightest neutralino as a dark matter candidate and include the WMAP upper constraint on its relic abundance [99] as well as the upper bound on the spin-independent neutralino-nucleon scattering cross section, $\sigma^{S I}$, from XENON100 [100]. The neutralino relic density and its scattering cross section are computed through an interface with Micromegas $[144,145]$. We have also modified the code NMSSMTOOLS to include three-body Higgs decay in the case of the NMSSM.

We have calculated the theoretical predictions for $\operatorname{BR}\left(B_{s}^{0} \rightarrow \mu^{+} \mu^{-}\right)$and compared them with the experimental value. In the case of the MSSM, the SUSY contributions to this observable [146] are likely to exceed the recent $\mathrm{LHCb}$ measurement $\mathrm{BR}\left(B_{s}^{0} \rightarrow \mu^{+} \mu^{-}\right)=$ $3.2_{-1.2}^{+1.5} \times 10^{-9}$ [92], especially for light pseudoscalars in the large $\tan \beta$ regime. In the case of the NMSSM [147-149], the corresponding Wilson coefficient receives contributions from both pseudoscalar Higgs bosons through their doublet components. Given that the light pseudoscalar is a purely singlet-like field, it leads to a negligible effect on this observable, and since $a_{2}^{0}$ is heavy enough and $\tan \beta$ is small, the experimental constraint is easily fulfilled. On the other hand, in general, $\operatorname{BR}(b \rightarrow s \gamma)$ provides a strong constraint on SUSY models. On top of the usual MSSM terms, NMSSM-specific contributions arise from the extended Higgs and the neutralino sectors, which come into effect at the two-loop level $[147,148]$. This observable has been shown to lead to stringent constraints on the NMSSM parameter space [57] and this is indeed the case in our current analysis. Here, we consider the experimental result [93] and include the theoretical error of the calculation in the SM $[150,151]$ in quadrature.

The SUSY contribution to the muon anomalous magnetic moment, $a_{\mu}^{\mathrm{SUSY}}$ was also computed. The observed discrepancy between the experimental value [94, 95] and the SM predictions using $e^{+} e^{-}$data favours positive contributions from new physics in the range $10.1 \times 10^{-10}<a_{\mu}^{\text {SUSY }}<42.1 \times 10^{-10}$ at the $2 \sigma$ confidence level [96-98], combining experimental and theoretical errors in quadrature. If tau data is employed, this discrepancy is smaller, $2.9 \times 10^{-10}<a_{\mu}^{\mathrm{SUSY}}<36.1 \times 10^{-10}[97]$.

We have carried out a simple scan to sample the NMSSM parameter space, searching for regions in the parameter space where Scenarios I and II could be realised. Out of the viable regions we have selected two benchmark points, BP1 and BP2 with parameters given in table 1, which constitute representative examples of Scenario I and Scenario II, 


\begin{tabular}{|c|c|c|}
\hline Parameter & BP1 & BP2 \\
\hline $\tan \beta$ & 5 & 5 \\
$\lambda, \kappa$ & $0.285,0.1165$ & $0.286,0.0844$ \\
$A_{\lambda}, A_{\kappa}(\mathrm{GeV})$ & $670,14.0$ & $820,14.35$ \\
$M_{\widetilde{L}_{i}}, M_{\widetilde{e}_{i}^{c}}(\mathrm{GeV})$ & 300,300 & 300,300 \\
$M_{\widetilde{Q}_{i}}, M_{\widetilde{u}_{i}^{c}}, M_{\widetilde{d}_{i}^{c}}(\mathrm{GeV})$ & $1000,1000,1000$ & $1000,1000,1000$ \\
$\mu(\mathrm{GeV})$ & 123.5 & 123.5 \\
$M_{1}, M_{2}, M_{3}(\mathrm{GeV})$ & $560,1200,1980$ & $240,500,1380$ \\
$A_{\tau}, A_{b}, A_{t}(\mathrm{GeV})$ & $-1600,1000,1800$ & $-1600,1000,1300$ \\
\hline
\end{tabular}

Table 1. Model parameters that define our choice of benchmark points. The top-quark pole mass is set to $173.5 \mathrm{GeV}$ and $m_{b} \overline{\mathrm{MS}}\left(m_{b}\right)=4.18 \mathrm{GeV}$.

\begin{tabular}{|c|c|c|}
\hline Parameter & BP1 & BP2 \\
\hline$m_{h_{1}^{0}}, m_{h_{2}^{0}}, m_{h_{3}^{0}}$ & $97.7,125.9,677.0$ & $62.1,125.9,739.8$ \\
$m_{a_{1}^{0}}, m_{a_{2}^{0}}$ & $3.6,675.4$ & $7.6,738.6$ \\
$m_{h^{ \pm}}, m_{\widetilde{\chi}_{1}^{ \pm}}, m_{\widetilde{\chi}_{2}^{ \pm}}$ & $679.8,124.0,1192$ & $739,118.1,522.3$ \\
$m_{\widetilde{\chi}_{1}^{0}}, m_{\widetilde{\chi}_{2}^{0}}, m_{\widetilde{\chi}_{3}^{0}}$ & $87.0,136.1,143.3$ & $63.6,124.8,139.0$ \\
$m_{\widetilde{t}_{1}}, m_{\widetilde{t}_{2}}, m_{\widetilde{b}_{1}}, m_{\widetilde{b}_{2}}$ & $859.5,1154,1008,1009$ & $951.7,1139,1040,1041$ \\
$m_{\widetilde{\tau}_{1}}, m_{\widetilde{\tau}_{2}}$ & $296.5,309.5$ & $296.5,309.4$ \\
$m_{\widetilde{g}}$ & 1926 & 1393 \\
\hline
\end{tabular}

Table 2. Relevant mass spectrum for the chosen benchmark points. For $m_{h_{2}^{0}}$ the latest ATLAS limit is $125.2 \pm 0.3$ (stat) \pm 0.6 (syst) GeV [154]. The CMS limit corresponds to $125.3 \pm 0.4$ (stat) \pm 0.5 (syst) [2]. Masses are given in GeV.

respectively. The squarks and gluino masses for the first two generation are chosen to be heavy enough to be in agreement with current LHC SUSY searches [152, 153]. We have relaxed gaugino mass unification in order to have more freedom in the neutralino composition, which is important in order to fix its relic abundance and scattering cross section. Also, trilinear parameters are non-universal and chosen in such a way that $a_{\mu}^{\mathrm{SUSY}}$ is maximized and $\mathrm{BR}(b \rightarrow s \gamma)$ is in agreement with the experimental value. The resulting mass spectra are shown in table 2 .

The compositions of Higgses, neutralinos, and charginos for our benchmark points are shown in table 3 . Since the $\mu$ parameter is chosen to be small and $\lambda \sim 0.3$, the singlet VEV is around $430 \mathrm{GeV}$. Thus with the small $\kappa$ value, the singlino mass, $2 \kappa v_{s}$ for the benchmark points BP1 and BP2 is approximately 72 and $100 \mathrm{GeV}$, respectively. Notice that this value is close to that of the $\mu$ parameter and that both of them are smaller than the bino and wino mass terms. This results in an interesting hierarchical neutralino spectrum, especially for the three lightest neutralino states. The lightest neutralino is a singlino-Higgsino state (where the singlino component slightly dominates). There is an orthogonal eigenstate which is also singlino-Higgsino (but this one with a larger Higgsino component), which in BP1 corresponds to the third neutralino and in BP2 is the second, 


\begin{tabular}{|c|c|c|}
\hline Mass eigenstate & BP1 $(\%)$ & BP2 $(\%)$ \\
\hline$h_{1}^{0}\left(S, H_{u}, H_{d}\right)$ & $79.9,17.6,2.5$ & $81.2,16.5,2.3$ \\
$h_{2}^{0}\left(S, H_{u}, H_{d}\right)$ & $19.5,78.2,2.3$ & $18.3,79.4,2.3$ \\
$h_{3}^{0}$ & $95.2 \% H_{d}$-like & $95.4 \% H_{d}$-like \\
$a_{1}^{0}$ & $99.6 \%$ singlet-like & $99.5 \%$ singlet-like \\
$a_{2}^{0}$ & $95.8 \% H_{d}$-like & $95.6 \% H_{d}$-like \\
$\widetilde{\chi}_{1}^{0}\left(\widetilde{B}^{0}, \widetilde{W}_{3}^{0}, \widetilde{H}_{d}, \widetilde{H}_{u}, \widetilde{S}\right)$ & $0.3,0.2,13.3,30.0,56.2$ & $1.7,0.9,7.8,22.7,66.9$ \\
$\widetilde{\chi}_{2}^{0}($ Higgsino, singlino $)$ & $96.8,2.9$ & $64.0,29.5$ \\
$\widetilde{\chi}_{3}^{0}$ (Higgsino, singlino $)$ & $58.8,40.9$ & $95.6,3.6$ \\
$\widetilde{\chi}_{1}^{ \pm}$ & $>95 \%$ Higgsino-like & $>95 \%$ Higgsino-like \\
\hline
\end{tabular}

Table 3. Compositions of Higges, neutralinos, and charginos for the chosen benchmark points.

\begin{tabular}{|c|c|c|c|}
\hline Quantity & BP1 & BP2 & Range/Limit \\
\hline $\mathrm{BR}(b \rightarrow s \gamma) \times 10^{4}$ & 3.86 & 3.69 & $2.86-4.24(2 \sigma)[93]$ \\
$\mathrm{BR}\left(B_{s} \rightarrow \mu^{+} \mu^{-}\right) \times 10^{9}$ & 3.97 & 3.63 & $2.0-4.7[92]$ \\
$\mathrm{BR}\left(B^{+} \rightarrow \tau^{+} \nu_{\tau}\right) \times 10^{4}$ & 1.31 & 1.31 & $0.85-2.89(2 \sigma)[155]$ \\
$R_{\gamma \gamma}$ & 1.0295 & 1.0385 & $1.80 \pm 0.50[\mathrm{ATLAS}][1,3,4]$ \\
& & $1.48_{-0.39}^{+0.54}[\mathrm{CMS}][2,5,6]$ \\
$a_{\mu}^{\mathrm{SUSY}} \times 10^{10}$ & 3.134 & 6.90 & $2.9-36.1(2 \sigma)[97]$ \\
& & $10.1-42.1(2 \sigma)[98]$ \\
Relic density $\left(\Omega_{\widetilde{\chi}_{1}^{0}} h^{2}\right)$ & 0.082 & 0.102 & $0.094-0.136[\mathrm{WMAP}][99]$ \\
$\sigma^{\mathrm{SI}} \times 10^{9}(\mathrm{pb})$ & 1.011 & 0.723 & $\lesssim 1.44 @ 86.99(2 \sigma)(\mathrm{BP} 1)[100]$ \\
& & & $\lesssim 1.17 @ 63.6(2 \sigma)(\mathrm{BP} 2)[100]$ \\
\hline
\end{tabular}

Table 4. Low-energy observables for the chosen benchmark point. When scanning the parameter space, we set $R_{\gamma \gamma} \equiv \frac{\sigma\left(g g \rightarrow h_{2}^{0} \rightarrow \gamma \gamma\right)}{\sigma\left(g g \rightarrow h_{S M} \rightarrow \gamma \gamma\right)}>0.8[24,25]$, within the $2 \sigma$ range of the ATLAS and the CMS result $[1-6]$. For $\mathrm{BR}(b \rightarrow s \gamma)$, the theoretical error is added to the experimental one in quadrature.

and close in mass to this one we can find an almost pure Higgsino $\left(\widetilde{\chi}_{2}^{0}\right.$ for BP1 and $\widetilde{\chi}_{3}^{0}$ for BP2). The heavier states are bino and wino-like.

The resulting predictions for low-energy observables are shown in table 4. Although the values of $a_{\mu}^{\text {SUSY }}$ are in the $2 \sigma$ range of the result from tau data, there is some tension with the one from $e^{+} e^{-}$data. We have tried reducing this discrepancy by decreasing the masses in the slepton sector. Regarding the neutralino relic abundance, it is well known that a Higgsino-like neutralino generally entails a large annihilation cross section and consequently a small relic abundance, but in our case, the presence of a sizable (slightly dominant as we said above) singlino component is welcome to make it compatible with the upper constraint on the dark matter relic density. Similarly, the elastic scattering cross section for neutralinos with a large Higgsino component is large and can be in conflict with current experimental bounds. In our case, the recent constraints from XENON100 are very severe and exclude a large portion of the parameter space we analyzed. The theoretical predictions for the spin-independent neutralino-nucleon scattering cross section in both $\mathrm{BP} 1$ and BP2 are below the current exclusion line which, for a mass in the range $60-$ 
$100 \mathrm{GeV}$, is of the order of $\lesssim 10^{-9} \mathrm{pb}$. Notice in this sense that increasing the singlino component through the decrease, e.g., of the $\kappa$ parameter may be useful to avoid this problem but this alternative might be questionable since it leads to a reduction in the di-photon production from the Higgs decay.

\section{Collider analysis}

Let us finally present the collider studies for the $4 \ell+\mathbb{E}_{\mathrm{T}}$ signal of the benchmarks shown in tables 1 and 2. As discussed in section 2, the benchmark points can be classified in terms of the Higgs masses, namely,

- Scenario I: $\left(m_{h_{2}^{0}}, m_{h_{1}^{0}}, m_{a_{1}^{0}}\right) \equiv(126,98,3.6) \mathrm{GeV}$ and

- Scenario II: $\left(m_{h_{2}^{0}}, m_{h_{1}^{0}}, m_{a_{1}^{0}}\right) \equiv(126,62,7.6) \mathrm{GeV}$.

In these benchmark points, $\operatorname{BR}\left(h_{2}^{0} \rightarrow a_{1}^{0} a_{1}^{0}\right)$ is comparable to or larger than $\operatorname{BR}\left(h_{2}^{0} \rightarrow Z Z^{*}\right)$, and $\operatorname{BR}\left(h_{1}^{0} \rightarrow a_{1}^{0} a_{1}^{0}\right) \sim 1$. The light pseudoscalar boson $a_{1}^{0}$ decays mainly into the di-tau final state with a branching fraction of $\sim 79$ (92) \% in Scenario I (II). Given that the pseudoscalar mass is larger than $2 m_{\tau}$, the di-muon decay mode is negligible in Scenario II, while it remains sub-leading for Scenario I. Thus, restating the expression (2.4), the leading final state will be

$$
h_{1,2}^{0} \rightarrow a_{1}^{0} a_{1}^{0} \rightarrow \tau^{+} \tau^{-} \tau^{+} \tau^{-} \rightarrow 2 \ell^{+}+2 \ell^{-}+\mathscr{E}_{\mathrm{T}},
$$

with $\ell=e, \mu$, or $\tau_{h}$. Here, electrons and muons are coming from leptonic tau decays, while $\tau_{h}$ denotes the tau jet originated from hadronic tau decays. Occasional muons can come from $a_{1}^{0} \rightarrow \mu^{+} \mu^{-}$decay process, which is sub-leading or negligible in the chosen benchmarks. The source of the missing energy is associated with neutrinos resulting from tau decays. In order to determine the feasibility of the signal at the LHC run with the $8 \mathrm{TeV}$ center-of-mass energy $(\sqrt{s})$, which has recently finished its operation, we consider inclusive search channels. In other words, our analysis differs from past studies which usually consider one or two exclusive channels such as $4 \mu$ or $2 \mu+2 \tau_{h}$ with the $14 \mathrm{TeV}$ beam condition.

We have generated Monte Carlo (MC) event samples of the Higgs signals for a protonproton collision at $\sqrt{s}=8 \mathrm{TeV}$ using HeRwIG $++2.6 .1[156,157]$ with the CTEQ6L1 parton distribution function (PDF) [158]. $h_{2}^{0} \rightarrow Z Z^{*} \rightarrow 4 \ell$ processes are also generated since the final states are similar as in eq. (4.1). The generated event samples have been scaled to $25 \mathrm{fb}^{-1}$ of integrated luminosity, which corresponds to the LHC data accumulated in the year 2012. To simplify the analysis, we here consider leading-order cross sections for all the processes. The production cross section and the decay width of the SM Higgs boson are calculated with HIGLU [159], then the NMSSM Higgs cross sections have been obtained by corrections according to the total decay widths and $\mathrm{BR}\left(h_{1,2}^{0} \rightarrow g g\right)$ calculated with NMSSMTOOLS. The generator-level events are further processed with the fast detector simulation program DeLPHES 2.0.3 [160] using a modified CMS detector card. In the detector simulation, jets are reconstructed by using the anti- $k_{t}$ algorithm [161] with the 
radius parameter of 0.5 , and the $b$-tagging efficiency is set to be $70 \%$. We assumed that the mis-tagging rate for c-jet is 10\%, and that for the gluon and light-flavor jets is $1 \%$, both of which are taken into account by Delphes. A candidate jet must satisfy $p_{\mathrm{T}}>25 \mathrm{GeV}$ for its transverse momentum and $|\eta|<2.5$ for its pseudorapidity. Isolated electrons and muons are required to have $p_{\mathrm{T}}>8$ and $6 \mathrm{GeV}$, respectively, and $|\eta|<2.4$. A tau jet is accepted only when it has $p_{\mathrm{T}}>15 \mathrm{GeV}$. In order to increase the purity of the leptonic signal, any charged lepton, including the tau jet, lying within a distance $\Delta R_{\ell j}<0.4$ from a candidate jet is discarded in the analysis, where $\Delta R_{a b}$ is defined as $\sqrt{\left(\eta_{a}-\eta_{b}\right)^{2}+\left(\phi_{a}-\phi_{b}\right)^{2}}$.

The dominant SM backgrounds include Drell-Yan (DY), $b \bar{b}, c \bar{c}$, semi- or di-leptonically decaying $t \bar{t}, W W / Z$, and $Z Z / \gamma$ processes. Direct $J / \psi$ or $\Upsilon$ productions can in principle contribute, but they are found to be almost negligible. To estimate the backgrounds, $b \bar{b}$ and $c \bar{c}$ event samples are generated with PYTHIA 6.4 [162] using CTEQ6L1 PDF in the various $p_{\mathrm{T}}$ bins, and the remaining dominant background processes are generated by HeRWIG ++ at the matrix-element level. The parton showering and the hadronization are performed by Herwig ++ for all the background processes. Then, the MC samples have been fed into DeLPHes using the same detector card used for the Higgs signals. In order to suppress the backgrounds while keeping the significance of the Higgs signals of interest, the following basic event selection cuts are imposed.

- At least two pairs of oppositely-charged leptons including the tau jet,

- no $b$-tagged jet.

When selecting the leptons, the priority is given to the hard electrons and isolated muons since the reconstruction efficiency of the tau jet is relatively poor. No cut is applied on the missing energy, despite the presence of neutrinos in the final state from tau decays. In fact, the neutrino momenta are approximately collinear with their parent tau momentum, leading to a partial cancellation among them and resulting in a quite small missing energy for signal events. To illustrate this, the signal distribution of the missing energy is compared to some dominant background distributions in the left panel of figure 1. As already mentioned in the previous section, multi-lepton and missing energy signatures of SUSY cascade decay processes (not included here) can also be serious backgrounds to the Higgs signal. However, the missing energy in these processes is expected to be much larger and one might attempt to use an upper cut on $\mathbb{E}_{\mathrm{T}}$. In any case, as we will see below, all these backgrounds can be efficiently suppressed by employing other cut variables.

For the events that passed the basic selection cuts, the combinatorial ambiguity of pairing the leptons is resolved by calculating all the possible $M_{\mathrm{T} 2}$ defined as in ref. [86, 87],

$$
M_{\mathrm{T} 2} \equiv \min _{\mathbf{k}_{\mathrm{T}}^{(1)}+\mathbf{k}_{\mathrm{T}}^{(2)}=\not \mathfrak{\mathrm { T }}_{\mathrm{T}}}\left[\max \left\{M_{\mathrm{T}}^{(1)}\left(\mathbf{p}_{\mathrm{T}}^{(1)}, \mathbf{k}_{\mathrm{T}}^{(1)}\right), M_{\mathrm{T}}^{(2)}\left(\mathbf{p}_{\mathrm{T}}^{(2)}, \mathbf{k}_{\mathrm{T}}^{(2)}\right)\right\}\right],
$$

where $M_{\mathrm{T}}^{(i)}(i=1,2)$ are the transverse masses calculated by the measured transverse momenta $\mathbf{p}_{\mathrm{T}}^{(i)}$ of the charged leptons and the invisible momenta $\mathbf{k}_{\mathrm{T}}^{(i)}$ of the neutrino system, which are determined by a numerical minimization over all possible splittings that maintain the missing energy condition constructed in the event. The $M_{\mathrm{T} 2}$ variable was introduced 

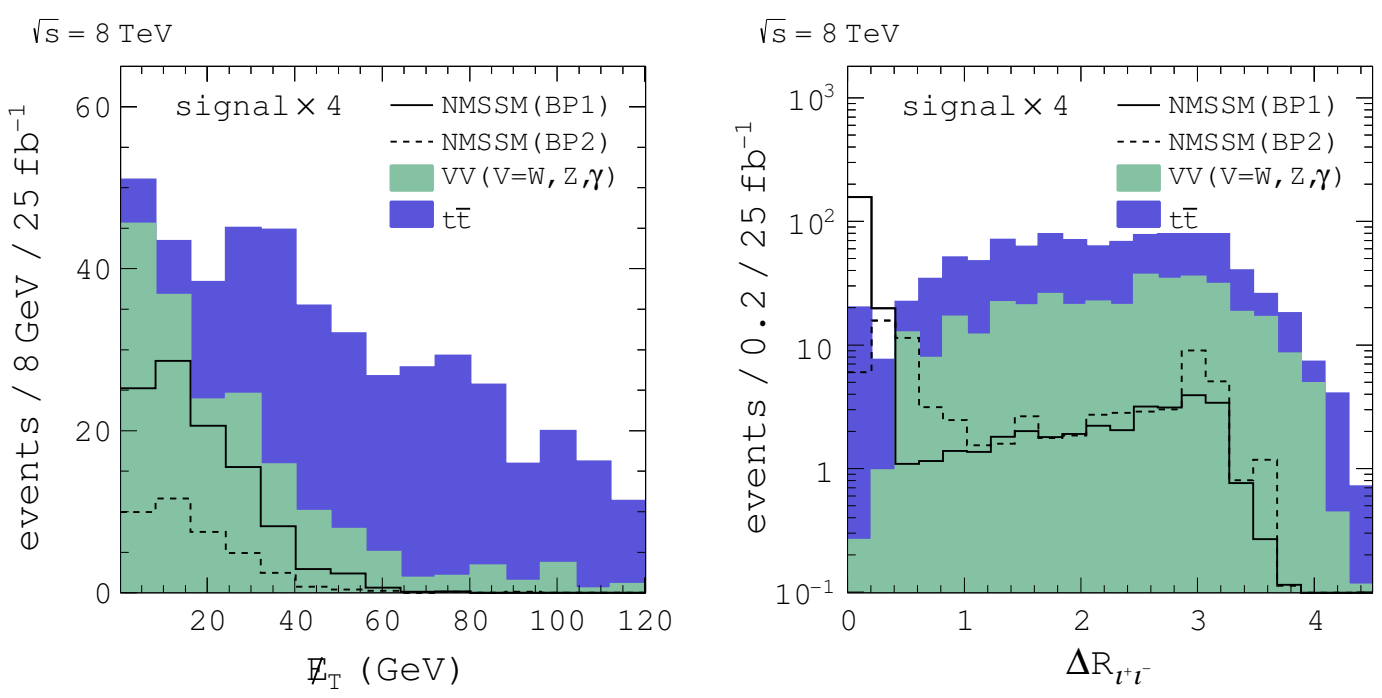

Figure 1. Distributions of (left panel) the missing energy and (right panel) $\Delta R_{\ell^{+} \ell^{-}}$for signals and some dominant backgrounds. The basic selection cuts have been imposed.

for a decay topology like a pair production of the squark that decays into a quark and the LSP. The decay topology given in expression (4.1) is essentially different from that because there are at least four neutrinos in each event. However, the $M_{\mathrm{T} 2}$ is applicable to the type of Higgs signal events considered here since the pseudoscalar bosons are emitted back-toback in the rest frame of the scalar Higgs boson. Thus their decay products are nearly collinear and the neutrinos sharing the same parent pseudoscalar boson can be considered as one invisible particle. In the construction of the $M_{\mathrm{T} 2}$, there are two possible ways of pairing the leptons because two pairs of oppositely-charged leptons are initially selected. We choose the pairing which gives the smaller value of $M_{\mathrm{T} 2}$. This method can be justified by the fact that the $M_{\mathrm{T} 2}$ value would be bounded from above by the parent particle mass $m_{a_{1}^{0}}$ if the right pairing is chosen, whereas there is no such a restriction if the pairing was wrong $[163,164]$. Since we here consider a light pseudoscalar boson, an upper cut on the $M_{\mathrm{T} 2}<25 \mathrm{GeV}$ is further implemented. The $M_{\mathrm{T} 2}$ distribution is shown in figure 2 for both benchmark points. We can appreciate a very clear peak and the endpoint structure around the pseudoscalar mass for both scenarios.

After choosing the pairing of the leptons, the transverse momentum $p_{\mathrm{T}}^{\ell^{+} \ell^{-}} \equiv\left|\mathbf{p}_{\mathrm{T}}^{\ell^{+}}+\mathbf{p}_{\mathrm{T}}^{\ell^{-}}\right|$ and the angular separation $\Delta R_{\ell^{+} \ell^{-}}$are calculated for each lepton pair. It is likely that the leptons coming from signal events are fairly boosted in the nearly-collinear direction. This can be observed in the right panel of figure 1, and provides a clear criterion for discriminating the signal and background. Motivated by this, we include selection cuts on the $p_{\mathrm{T}}^{\ell^{+} \ell^{-}}$and $\Delta R_{\ell^{+} \ell^{-}}$as follows,

- $p_{\mathrm{T}}^{\ell^{+} \ell^{-}}>15 \mathrm{GeV}$,

- $\min \left\{\Delta R_{\ell^{+} \ell^{-}}^{(1)}, \Delta R_{\ell^{+} \ell^{-}}^{(2)}\right\}<0.12$ or $\Delta R_{\ell^{+} \ell^{-}}<0.65$ for both pairs. 

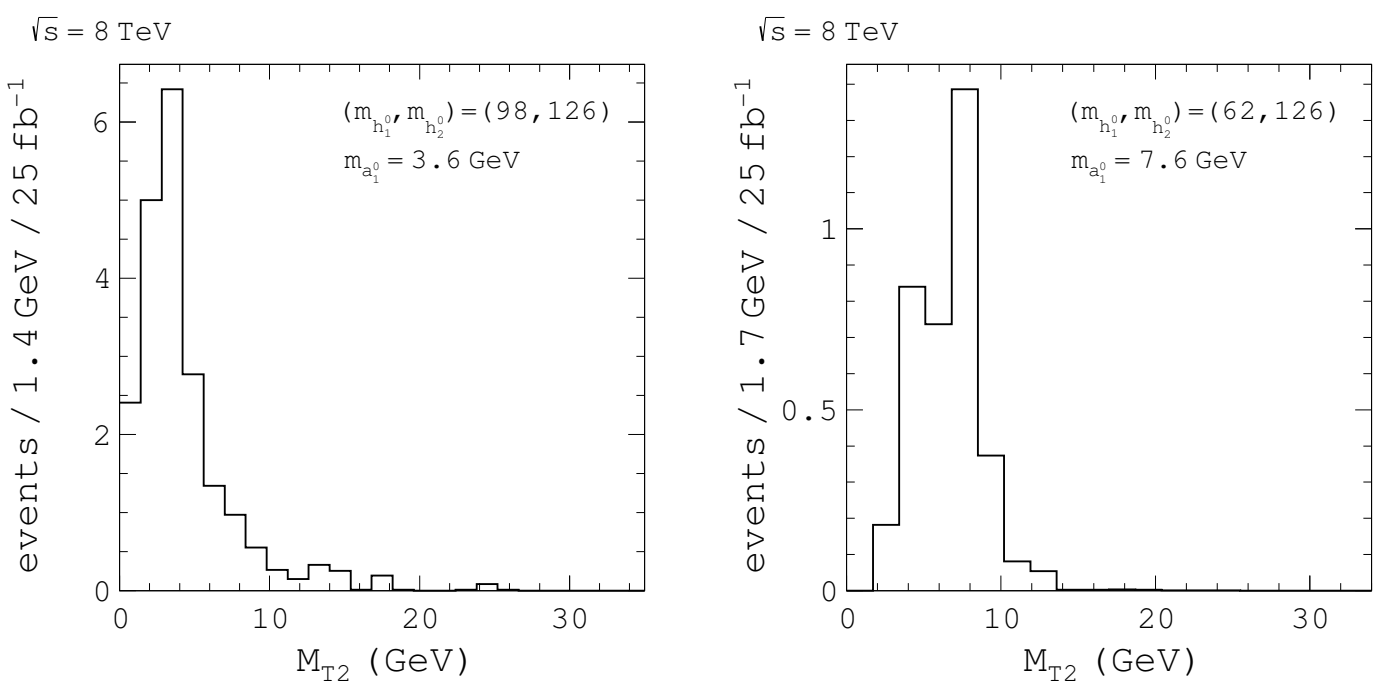

Figure 2. The $M_{\mathrm{T} 2}$ distributions of four visible leptons + missing energy signals for (left panel) the BP1 and (right panel) the BP2. All the event selection cuts described in the text except the jet-veto condition have been imposed.

An important observation is that after applying the above cuts, the $h_{2}^{0} \rightarrow Z Z^{*} \rightarrow 2 \ell^{+} 2 \ell^{-}$ background is found to be almost negligible since the collinearity of the leptons is not valid for such kind of events. On top of that, the invariant masses of the oppositely-charged lepton pairs are required to be $m_{\ell^{+} \ell^{-}}<20 \mathrm{GeV}$ to ensure that the lepton pairs come from the light pseudoscalar. Collectively, a low $\Delta R_{\ell^{+} \ell^{-}}$together with a low $m_{\ell^{+} \ell^{-}}$cut can discriminate the studied signal from the $h_{2}^{0} \rightarrow Z Z^{*} \rightarrow 2 \ell^{+} 2 \ell^{-}$process.

We also calculate the cluster transverse mass defined as

$$
M_{\mathrm{T}}^{2} \equiv\left(\sqrt{m_{\mathcal{V}}^{2}+\left|\mathbf{p}_{\mathrm{T}}^{\mathcal{V}}\right|^{2}}+\mathbb{E}_{\mathrm{T}}\right)^{2}-\left|\mathbf{p}_{\mathrm{T}}^{\mathcal{V}}+\mathbf{p}_{\mathrm{T}}\right|^{2}
$$

where $\mathcal{V}$ denotes the four-lepton system [165]. The endpoint position of the $M_{\mathrm{T}}$ distribution corresponds to the parent particle mass of the pseudoscalar bosons, i.e., $m_{h_{1}^{0}}$ or $m_{h_{2}^{0}}$ if there exists an event with vanishing invariant mass of the neutrinos and all the particle tracks are on the transverse plane. However, one cannot distinguish the decay event of $h_{1}^{0}$ from that of $h_{2}^{0}$ as they lead to the same final states. The $M_{\mathrm{T}}$ distributions are shown in figure 3. For both scenarios, the signal distributions are largely populated around/below $m_{h_{1}^{0}}$, whereas no clear peak structure is observed around $m_{h_{2}^{0}}$. The main reason is the different production cross sections of $h_{1}^{0}$ and $h_{2}^{0}$. At the generator level we obtain $\sigma_{g g \rightarrow h_{1}^{0} \rightarrow 2 a_{1}^{0} \rightarrow 4 \ell} / \sigma_{g g \rightarrow h_{2}^{0} \rightarrow 2 a_{1}^{0} \rightarrow 4 \ell} \sim 7$, and this ratio is still as large as $\sim 4$ in the detector-level data when applying the basic selection cuts to the triggered events in the case of the BP1. ${ }^{5}$ For the same reason, the $M_{\mathrm{T}}$ distribution of the BP2 has a clear peak around the $m_{h_{1}^{0}}$ value as well. Although the contribution from the small $h_{2}^{0}$ decay events

\footnotetext{
${ }^{5}$ The change of the ratio is due to the fact that the light Higgs boson leads to relatively soft final-state leptons, and consequently, the acceptance at the detector becomes poor.
} 

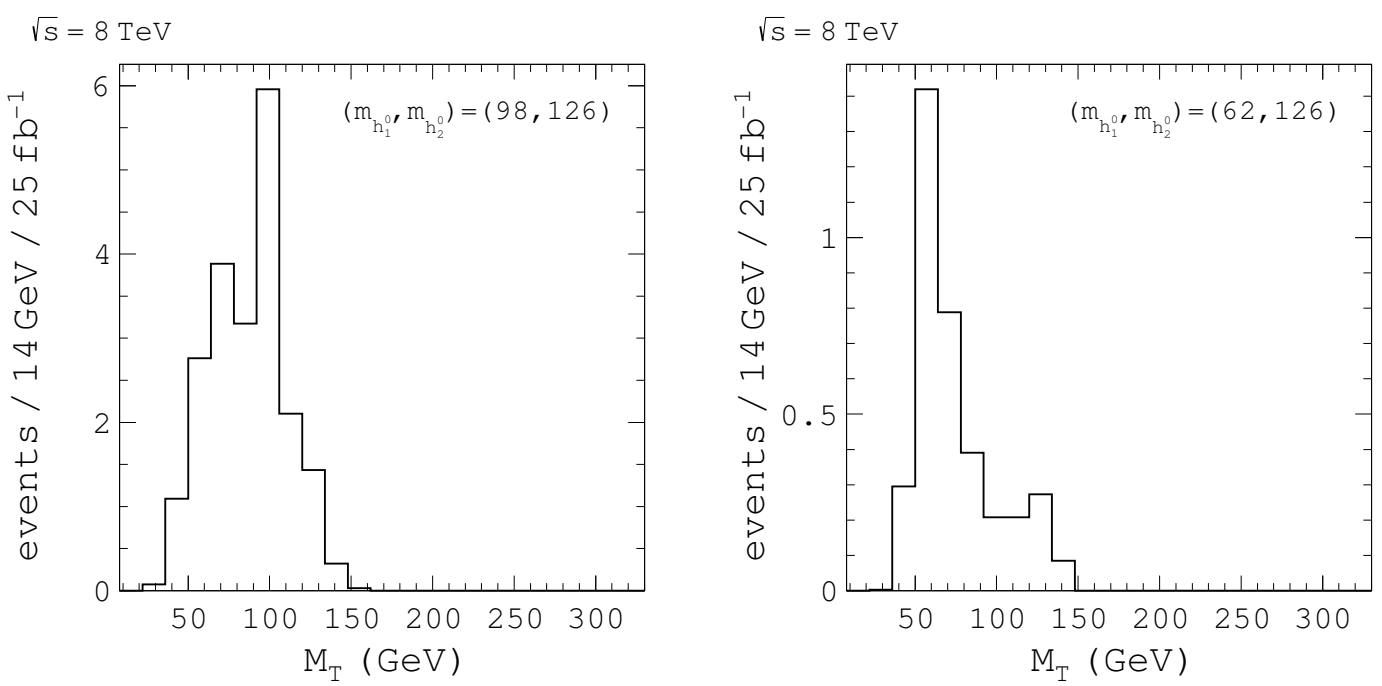

Figure 3. Transverse mass distributions of four visible leptons of the signals for (left panel) the BP1 and (right panel) the BP2. All the event selection cuts described in the text except the jet-veto condition have been imposed.

smears out the edge of the $M_{\mathrm{T}}$ distribution, one can still estimate the light Higgs boson mass scale by the peak position.

The $M_{\mathrm{T}}$ variable can also be used to define the signal region. We consider the events with $35<M_{\mathrm{T}}<145 \mathrm{GeV}$, which corresponds to the region where two light Higgs bosons can be discovered. This definition of the signal region might be useful if there exists a new physics process whose final state contains several leptons and massive invisible particles. Even though the new processes can contribute to the background of the Higgs signal, they can be readily isolated since the $M_{\mathrm{T}}$ values of such processes are generically large.

For events passing all the selection cuts, we also construct the invariant mass of all visible leptons, $m_{2 \ell^{+} 2 \ell^{-}}$. The peak position of the invariant mass distribution is generically different from $m_{h_{1}^{0}}$ and $m_{h_{2}^{0}}$ due to invisible neutrinos from the tau decays. We impose a cut, requiring the invariant mass to be larger than $40 \mathrm{GeV}$, which accommodates to the possible spread of the invariant mass distribution. Another possible collider variable is the invariant mass reconstructed by the $M_{\mathrm{T} 2}$-assisted on-shell approximation of the invisible momenta [166], which has been found to be useful for reconstructing the resonance mass peak in the similar decay topology [167-169]. However, by definition, we would need to sacrifice a certain signal statistics by imposing a strong $M_{\mathrm{T} 2}$ cut to attain a good accuracy. We expect that this variable would be useful with higher luminosity.

After imposing all the event selection cuts, we find that all the considered SM backgrounds turn out to be completely negligible. In order to estimate the signal significance, we show how the number of events of the Higgs signals and the backgrounds change under each event selection cut in table 5 . The cuts on $p_{\mathrm{T}}^{\ell^{+} \ell^{-}}$and $\Delta R_{\ell^{+} \ell^{-}}$are especially efficient in discriminating the signal from the otherwise dominant DY and $b \bar{b}$ backgrounds, resulting in a good statistical significance for the benchmark point BP1 and a poorer result for BP2. 


\begin{tabular}{|c|cc|rrrr|rr|}
\hline Selection cuts & BP1 & BP2 & DY & $b \bar{b}$ & $t \bar{t}$ & Diboson & $\hat{\mu}_{\mathrm{BP} 1}$ & $\hat{\mu}_{\mathrm{BP} 2}$ \\
\hline Basic cuts & 26.1 & 9.5 & 252519.8 & 7998.1 & 325.2 & 186.8 & 0.05 & 0.02 \\
$M_{\mathrm{T} 2}$ & 22.0 & 4.6 & 1233.6 & 3225.7 & 2.4 & 0.5 & 0.3 & 0.07 \\
$m_{\ell^{+} \ell^{-}}$ & 21.8 & 4.3 & 145.4 & 2258.2 & 1.2 & 0.2 & 0.4 & 0.09 \\
$p_{\mathrm{T}}^{\ell^{+}}, \Delta R_{\ell^{+} \ell^{-}}$ & 20.8 & 3.7 & 4.7 & 40.7 & 0.0 & 0.1 & 2.9 & 0.5 \\
$M_{\mathrm{T}}$ & 20.8 & 3.7 & 4.7 & 0.0 & - & 0.0 & 6.7 & 1.5 \\
$m_{2 \ell^{+} 2 \ell^{-}}$ & 20.1 & 3.4 & 0.0 & - & - & - & - & - \\
Jet veto & 19.2 & 3.2 & - & - & - & - & - & - \\
\hline
\end{tabular}

Table 5. Cut flow of the signals and backgrounds and corresponding signal significance calculated with the Poisson probabilities for BP1 and BP2 at the integrated luminosity of $25 \mathrm{fb}^{-1}$. See the text for the detailed descriptions of the cuts applied.

We define $\hat{\mu}$ as the statistical significance calculated with the Poisson probabilities since the number of background events is small when applying all the cuts. Indeed, the cut efficiency of each scenario is rather different. One reason seems to be the dissimilar efficiency of the object reconstructions. In $\mathrm{BP} 1$ the decay products of the pseudoscalar boson are relatively adjacent as it is likely that they are more boosted than in the case of BP2, and this can affect the correct identification of the final-state particles.

Notice finally that since there is no source of hard jets except for initial state radiation in the signal process, one can further impose a jet-veto condition that discards events containing hard jets and can be employed for suppressing rare processes like $t \bar{t}+$ jets or $W W+$ jets, which have not been included in this study. The (small) effect of this cut with $p_{\mathrm{T}}>50 \mathrm{GeV}$ on the signal, is listed in the last row for use in studies that consider the other possible backgrounds.

To summarise, our simulation and analysis establish that the current $8 \mathrm{TeV}$ data from the LHC, with dedicated selection cuts, can be used to test parts of the parameter space of non-standard Higgs scenarios with very light pseudoscalars.

\section{Conclusions}

In the light of the recent detection at the LHC of a scalar boson with a mass of $126 \mathrm{GeV}$ and the possible hint for an excess in LEP that could correspond to a second, lighter scalar we investigate the phenomenology and detectability of viable models in the NMSSM. We consider two scenarios that contain two light Higgses, in which the mass of the lighter scalar is either in the range of $96-100 \mathrm{GeV}$ or lighter than half of the SM-like Higgs mass. Both of them are analyzed in the presence of a very light pseudoscalar boson, $2 m_{\tau} \lesssim m_{a_{1}^{0}}<2 m_{b}$, a range which is yet to be explored further at the LHC. The Higgs phenomenology of these scenarios is extremely rich, involving cascade Higgs decays that can lead up to eight 
leptons in the final state, with or without missing energy. We perform a reconstruction of these channels for two representative benchmark points, determining the optimal cuts that allow us to single out the signal from the background. We show that the pseudoscalar mass can be successfully determined from the $M_{\mathrm{T} 2}$ distribution of four visible leptons and the missing energy. We also investigate the reconstruction of the light scalar Higgs boson from the $M_{\mathrm{T}}$ of the four visible leptons and the missing energy. The Higgs boson mass can be estimated by the peak position of the $M_{\mathrm{T}}$ distribution. Our analysis suggests that the experimental search for inclusive decay modes using the current $8 \mathrm{TeV}$ LHC data and dedicated cuts can be used to test parts of the parameter space on these possible two-Higgs scenarios with very light pseudoscalars.

\section{Acknowledgments}

The work of D.G. Cerdeño and P. Ghosh is supported in part by the Spanish MINECO under grants FPA2009-08958, FPA2009-09017 and FPA2012-34694, and under the 'Centro de Excelencia Severo Ochoa' Programme SEV-2012-0249, by the Comunidad de Madrid under grant HEPHACOS S2009/ESP-1473, and by the European Union under the Marie Curie-ITN program PITN-GA-2009-237920. The work of C.B. Park is supported by the CERN-Korea fellowship through National Research Foundation of Korea. C. B. Park gratefully acknowledges the hospitality of IFT UAM/CSIC, where part of this work was carried out.

Open Access. This article is distributed under the terms of the Creative Commons Attribution License which permits any use, distribution and reproduction in any medium, provided the original author(s) and source are credited.

\section{References}

[1] ATLAS collaboration, Observation of a new particle in the search for the Standard Model Higgs boson with the ATLAS detector at the LHC, Phys. Lett. B 716 (2012) 1 [arXiv:1207.7214] [INSPIRE].

[2] CMS collaboration, Observation of a new boson at a mass of $125 \mathrm{GeV}$ with the CMS experiment at the LHC, Phys. Lett. B $\mathbf{7 1 6}$ (2012) 30 [arXiv:1207.7235] [INSPIRE].

[3] ATLAS collaboration, Observation of an excess of events in the search for the Standard Model Higgs boson in the gamma-gamma channel with the ATLAS detector, ATLAS-CONF-2012-091 (2012).

[4] ATLAS collaboration, Updated ATLAS results on the signal strength of the Higgs-like boson for decays into $W W$ and heavy fermion final states, ATLAS-CONF-2012-162 (2012).

[5] CMS collaboration, Evidence for a new state decaying into two photons in the search for the standard model Higgs boson in pp collisions, CMS-PAS-HIG-12-015.

[6] CMS collaboration, Combination of standard model Higgs boson searches and measurements of the properties of the new boson with a mass near $125 \mathrm{GeV}$, CMS-PAS-HIG-12-045. 
[7] J. Baglio, A. Djouadi and R. Godbole, The apparent excess in the Higgs to di-photon rate at the LHC: New Physics or QCD uncertainties?, Phys. Lett. B 716 (2012) 203 [arXiv: 1207.1451] [INSPIRE].

[8] U. Ellwanger, A Higgs boson near $125 \mathrm{GeV}$ with enhanced di-photon signal in the NMSSM, JHEP 03 (2012) 044 [arXiv:1112.3548] [INSPIRE].

[9] R. Benbrik, M. Gomez Bock, S. Heinemeyer, O. Stal, G. Weiglein et al., Confronting the MSSM and the NMSSM with the Discovery of a Signal in the two Photon Channel at the LHC, Eur. Phys. J. C 72 (2012) 2171 [arXiv:1207.1096] [INSPIRE].

[10] J.F. Gunion, Y. Jiang and S. Kraml, Could two NMSSM Higgs bosons be present near 125 GeV?, Phys. Rev. D 86 (2012) 071702 [arXiv:1207.1545] [InSPIRE].

[11] J.F. Gunion, Y. Jiang and S. Kraml, The Constrained NMSSM and Higgs near $125 \mathrm{GeV}$, Phys. Lett. B 710 (2012) 454 [arXiv:1201.0982] [inSPIRE].

[12] S. King, M. Muhlleitner and R. Nevzorov, NMSSM Higgs Benchmarks Near $125 \mathrm{GeV}$, Nucl. Phys. B 860 (2012) 207 [arXiv:1201.2671] [INSPIRE].

[13] J.-J. Cao, Z.-X. Heng, J.M. Yang, Y.-M. Zhang and J.-Y. Zhu, A SM-like Higgs near $125 \mathrm{GeV}$ in low energy SUSY: a comparative study for MSSM and NMSSM, JHEP 03 (2012) 086 [arXiv:1202.5821] [InSPIRE].

[14] D.A. Vasquez, G. Bélanger, C. Boehm, J. Da Silva, P. Richardson et al., The $125 \mathrm{GeV}$ Higgs in the NMSSM in light of LHC results and astrophysics constraints, Phys. Rev. D 86 (2012) 035023 [arXiv:1203.3446] [INSPIRE].

[15] U. Ellwanger and C. Hugonie, Higgs bosons near $125 \mathrm{GeV}$ in the NMSSM with constraints at the GUT scale, Adv. High Energy Phys. 2012 (2012) 625389 [arXiv:1203.5048] [InSPIRE].

[16] K.J. Bae, K. Choi, E.J. Chun, S.H. Im, C.B. Park et al., Peccei-Quinn NMSSM in the light of $125 \mathrm{GeV}$ Higgs, JHEP 11 (2012) 118 [arXiv:1208.2555] [INSPIRE].

[17] G. Bélanger, U. Ellwanger, J. Gunion, Y. Jiang and S. Kraml, Two Higgs Bosons at the Tevatron and the LHC?, arXiv:1208.4952 [INSPIRE].

[18] I. Gogoladze, B. He and Q. Shafi, Inverse Seesaw in NMSSM and 126 GeV Higgs Boson, Phys. Lett. B 718 (2013) 1008 [arXiv: 1209.5984] [INSPIRE].

[19] K. Choi, S.H. Im, K.S. Jeong and M. Yamaguchi, Higgs mixing and diphoton rate enhancement in NMSSM models, JHEP 02 (2013) 090 [arXiv:1211.0875] [INSPIRE].

[20] K. Kowalska, S. Munir, L. Roszkowski, E.M. Sessolo, S. Trojanowski et al., The Constrained NMSSM with a $125 \mathrm{GeV}$ Higgs boson - A global analysis, arXiv:1211.1693 [INSPIRE].

[21] K. Schmidt-Hoberg, F. Staub and M.W. Winkler, Enhanced diphoton rates at Fermi and the LHC, JHEP 01 (2013) 124 [arXiv:1211.2835] [INSPIRE].

[22] J. Cao, Z. Heng, J.M. Yang and J. Zhu, Status of low energy SUSY models confronted with the LHC $125 \mathrm{GeV}$ Higgs data, JHEP 10 (2012) 079 [arXiv:1207.3698] [INSPIRE].

[23] J.F. Gunion, Y. Jiang and S. Kraml, Diagnosing Degenerate Higgs Bosons at $125 \mathrm{GeV}$, Phys. Rev. Lett. 110 (2013) 051801 [arXiv:1208.1817] [INSPIRE].

[24] G. Bélanger, U. Ellwanger, J.F. Gunion, Y. Jiang, S. Kraml et al., Higgs Bosons at 98 and $125 \mathrm{GeV}$ at LEP and the LHC, JHEP 01 (2013) 069 [arXiv: 1210.1976] [INSPIRE].

[25] S. King, M. Mühlleitner, R. Nevzorov and K. Walz, Natural NMSSM Higgs Bosons, Nucl. Phys. B 870 (2013) 323 [arXiv:1211.5074] [INSPIRE]. 
[26] Z. Kang, J. Li and T. Li, On Naturalness of the MSSM and NMSSM, JHEP 11 (2012) 024 [arXiv: 1201.5305] [INSPIRE].

[27] K. Agashe, Y. Cui and R. Franceschini, Natural Islands for a $125 \mathrm{GeV}$ Higgs in the scale-invariant NMSSM, JHEP 02 (2013) 031 [arXiv:1209.2115] [INSPIRE].

[28] U. Ellwanger, G. Espitalier-Noel and C. Hugonie, Naturalness and Fine Tuning in the NMSSM: Implications of Early LHC Results, JHEP 09 (2011) 105 [arXiv:1107.2472] [INSPIRE].

[29] A. Arvanitaki and G. Villadoro, A Non Standard Model Higgs at the LHC as a Sign of Naturalness, JHEP 02 (2012) 144 [arXiv:1112.4835] [INSPIRE].

[30] T. Cheng, J. Li, T. Li, X. Wan, Y.k. Wang et al., Toward the Natural and Realistic NMSSM with and without R-Parity, arXiv:1207.6392 [INSPIRE].

[31] M. Perelstein and B. Shakya, XENON100 Implications for Naturalness in the MSSM, NMSSM and lambda-SUSY, arXiv:1208.0833 [INSPIRE].

[32] M. Drees, Supersymmetric Models with Extended Higgs Sector, Int. J. Mod. Phys. A 4 (1989) 3635 [inSPIRE].

[33] J.R. Ellis, J. Gunion, H.E. Haber, L. Roszkowski and F. Zwirner, Higgs Bosons in a Nonminimal Supersymmetric Model, Phys. Rev. D 39 (1989) 844 [INSPIRE].

[34] P. Binetruy and C.A. Savoy, Higgs and top masses in a nonminimal supersymmetric theory, Phys. Lett. B 277 (1992) 453 [INSPIRE].

[35] J. Espinosa and M. Quirós, On Higgs boson masses in nonminimal supersymmetric standard models, Phys. Lett. B 279 (1992) 92 [INSPIRE].

[36] J. Espinosa and M. Quirós, Upper bounds on the lightest Higgs boson mass in general supersymmetric Standard Models, Phys. Lett. B 302 (1993) 51 [hep-ph/9212305] [INSPIRE].

[37] J.E. Kim and H.P. Nilles, The $\mu$ Problem and the Strong CP Problem, Phys. Lett. B 138 (1984) 150 [INSPIRE].

[38] U. Ellwanger, Enhanced di-photon Higgs signal in the Next-to-Minimal Supersymmetric Standard Model, Phys. Lett. B 698 (2011) 293 [arXiv:1012.1201] [INSPIRE].

[39] K. Schmidt-Hoberg and F. Staub, Enhanced $h \rightarrow \gamma \gamma$ rate in MSSM singlet extensions, JHEP 10 (2012) 195 [arXiv:1208.1683] [INSPIRE].

[40] J.F. Gunion, H.E. Haber and T. Moroi, Will at least one of the Higgs bosons of the next-to-minimal supersymmetric extension of the standard model be observable at LEP-2 or the LHC?, eConf C 960625 (1996) LTH095 [hep-ph/9610337] [INSPIRE].

[41] B.A. Dobrescu, G.L. Landsberg and K.T. Matchev, Higgs boson decays to CP odd scalars at the Tevatron and beyond, Phys. Rev. D 63 (2001) 075003 [hep-ph/0005308] [INSPIRE].

[42] U. Ellwanger, J.F. Gunion, C. Hugonie and S. Moretti, Towards a no lose theorem for NMSSM Higgs discovery at the LHC, hep-ph/0305109 [INSPIRE].

[43] U. Ellwanger, J. Gunion, C. Hugonie and S. Moretti, NMSSM Higgs discovery at the LHC, hep-ph/0401228 [INSPIRE].

[44] R. Dermisek and J.F. Gunion, Escaping the large fine tuning and little hierarchy problems in the next to minimal supersymmetric model and $h \rightarrow$ aa decays,

Phys. Rev. Lett. 95 (2005) 041801 [hep-ph/0502105] [INSPIRE]. 
[45] U. Ellwanger, J.F. Gunion and C. Hugonie, Difficult scenarios for NMSSM Higgs discovery at the LHC, JHEP 07 (2005) 041 [hep-ph/0503203] [INSPIRE].

[46] R. Dermisek and J.F. Gunion, Many Light Higgs Bosons in the NMSSM, Phys. Rev. D 79 (2009) 055014 [arXiv:0811.3537] [inSPIRE].

[47] R. Dermisek and J.F. Gunion, Direct production of a light CP-odd Higgs boson at the Tevatron and LHC, Phys. Rev. D 81 (2010) 055001 [arXiv:0911.2460] [INSPIRE].

[48] R. Dermisek and J.F. Gunion, New constraints on a light CP-odd Higgs boson and related NMSSM Ideal Higgs Scenarios, Phys. Rev. D 81 (2010) 075003 [arXiv:1002.1971] [INSPIRE].

[49] S. Abel, S. Sarkar and I. Whittingham, Neutralino dark matter in a class of unified theories, Nucl. Phys. B 392 (1993) 83 [hep-ph/9209292] [INSPIRE].

[50] U. Ellwanger and C. Hugonie, Topologies of the $(M+1)$ SSM with a singlino LSP at LEP-2, Eur. Phys. J. C 13 (2000) 681 [hep-ph/9812427] [INSPIRE].

[51] J.F. Gunion, D. Hooper and B. McElrath, Light neutralino dark matter in the NMSSM, Phys. Rev. D 73 (2006) 015011 [hep-ph/0509024] [INSPIRE].

[52] D. Das and U. Ellwanger, Light dark matter in the NMSSM: upper bounds on direct detection cross sections, JHEP 09 (2010) 085 [arXiv: 1007.1151] [INSPIRE].

[53] A.V. Belikov, J.F. Gunion, D. Hooper and T.M. Tait, CoGeNT, DAMA and Light Neutralino Dark Matter, Phys. Lett. B 705 (2011) 82 [arXiv:1009.0549] [INSPIRE].

[54] J.F. Gunion, A.V. Belikov and D. Hooper, CoGeNT, DAMA and Neutralino Dark Matter in the Next-To-Minimal Supersymmetric Standard Model, arXiv:1009.2555 [INSPIRE].

[55] D.A. Vasquez, G. Bélanger, C. Boehm, A. Pukhov and J. Silk, Can neutralinos in the MSSM and NMSSM scenarios still be light?, Phys. Rev. D 82 (2010) 115027 [arXiv: 1009.4380] [INSPIRE].

[56] D. Cerdeno, C. Hugonie, D. Lopez-Fogliani, C. Muñoz and A. Teixeira, Theoretical predictions for the direct detection of neutralino dark matter in the NMSSM, JHEP 12 (2004) 048 [hep-ph/0408102] [INSPIRE].

[57] D. Cerdeno, E. Gabrielli, D. Lopez-Fogliani, C. Muñoz and A. Teixeira, Phenomenological viability of neutralino dark matter in the NMSSM, JCAP 06 (2007) 008 [hep-ph/0701271] [INSPIRE].

[58] U. Ellwanger, J.F. Gunion and C. Hugonie, Establishing a no lose theorem for NMSSM Higgs boson discovery at the LHC, hep-ph/0111179 [INSPIRE].

[59] . Miller, D.J. and S. Moretti, An Interesting NMSSM scenario at the LHC and LC: A Contribution to the LHC/LC study group, hep-ph/0403137 [INSPIRE].

[60] S. Moretti, S. Munir and P. Poulose, Another step towards a no-lose theorem for NMSSM Higgs discovery at the LHC, Phys. Lett. B 644 (2007) 241 [hep-ph/0608233] [INSPIRE].

[61] R. Dermisek and J.F. Gunion, The NMSSM Close to the R-symmetry Limit and Naturalness in $h \rightarrow$ aa Decays for $m_{a}<2 m_{b}$, Phys. Rev. D 75 (2007) 075019 [hep-ph/0611142] [INSPIRE].

[62] K. Cheung, J. Song and Q.-S. Yan, Role of $h \rightarrow \eta \eta$ in Intermediate-Mass Higgs Boson Searches at the Large Hadron Collider, Phys. Rev. Lett. 99 (2007) 031801 [hep-ph/0703149] [INSPIRE].

[63] S. Chang, P.J. Fox and N. Weiner, Visible Cascade Higgs Decays to Four Photons at Hadron Colliders, Phys. Rev. Lett. 98 (2007) 111802 [hep-ph/0608310] [InSPIRE]. 
[64] J. Forshaw, J. Gunion, L. Hodgkinson, A. Papaefstathiou and A. Pilkington, Reinstating the 'no-lose' theorem for NMSSM Higgs discovery at the LHC, JHEP 04 (2008) 090 [arXiv:0712.3510] [INSPIRE].

[65] A. Belyaev, S. Hesselbach, S. Lehti, S. Moretti, A. Nikitenko et al., The Scope of the 4 tau Channel in Higgs-strahlung and Vector Boson Fusion for the NMSSM No-Lose Theorem at the $L H C$, arXiv:0805.3505 [INSPIRE].

[66] DELPHI collaboration, J. Abdallah et al., Searches for neutral Higgs bosons in extended models, Eur. Phys. J. C 38 (2004) 1 [hep-ex/0410017] [InSPIRE].

[67] R. Dermisek and J.F. Gunion, The NMSSM Solution to the Fine-Tuning Problem, Precision Electroweak Constraints and the Largest LEP Higgs Event Excess, Phys. Rev. D 76 (2007) 095006 [arXiv: 0705.4387] [INSPIRE].

[68] M. Carena, T. Han, G.-Y. Huang and C.E. Wagner, Higgs Signal for $h \rightarrow$ aa at Hadron Colliders, JHEP 04 (2008) 092 [arXiv:0712.2466] [INSPIRE].

[69] M.M. Almarashi and S. Moretti, Muon Signals of Very Light CP-odd Higgs states of the NMSSM at the LHC, Phys. Rev. D 83 (2011) 035023 [arXiv:1101.1137] [INSPIRE].

[70] M. Almarashi and S. Moretti, Very Light CP-odd Higgs bosons of the NMSSM at the LHC in 4b-quark final states, Phys. Rev. D 84 (2011) 015014 [arXiv:1105.4191] [INSPIRE].

[71] OPAL collaboration, G. Abbiendi et al., Search for a low mass CP odd Higgs boson in $e^{+} e^{-}$collisions with the OPAL detector at LEP-2, Eur. Phys. J. C 27 (2003) 483 [hep-ex/0209068] [INSPIRE].

[72] M. Lisanti and J.G. Wacker, Discovering the Higgs with Low Mass Muon Pairs, Phys. Rev. D 79 (2009) 115006 [arXiv:0903.1377] [INSPIRE].

[73] CMS collaboration, CMS Physics: Technical Design Report Volume 1: Detector Performance and Software, CERN-LHCC-2006-001 (2006).

[74] S.-h. Zhu, Unique Higgs boson signature at colliders, hep-ph/0611270 [INSPIRE].

[75] A. Belyaev, J. Pivarski, A. Safonov, S. Senkin and A. Tatarinov, LHC discovery potential of the lightest NMSSM Higgs in the $H 1 \rightarrow a 1 a 1 \rightarrow 4 \mu$ s channel, Phys. Rev. D 81 (2010) 075021 [arXiv:1002.1956] [INSPIRE].

[76] A. Martin, Higgs Cascade Decays to $\gamma \gamma+$ jet jet at the $L H C$, hep-ph/0703247 [INSPIRE].

[77] LeP Working Group for Higgs boson searches, ALEPH, DELPHI, L3, OPAL collaborations, R. Barate et al., Search for the standard model Higgs boson at LEP, Phys. Lett. B 565 (2003) 61 [hep-ex/0306033] [INSPIRE].

[78] Aleph, Delphi, L3, OPAl, LeP Working Group for Higgs Boson Searches collaborations, S. Schael et al., Search for neutral MSSM Higgs bosons at LEP, Eur. Phys. J. C 47 (2006) 547 [hep-ex/0602042] [INSPIRE].

[79] M. Drees, A Supersymmetric Explanation of the Excess of Higgs-Like Events at the LHC and at LEP, Phys. Rev. D 86 (2012) 115018 [arXiv:1210.6507] [INSPIRE].

[80] S. Heinemeyer, O. Stal and G. Weiglein, Interpreting the LHC Higgs Search Results in the MSSM, Phys. Lett. B 710 (2012) 201 [arXiv:1112.3026] [InSPIRE].

[81] P. Bechtle, S. Heinemeyer, O. Stal, T. Stefaniak, G. Weiglein et al., MSSM Interpretations of the LHC Discovery: Light or Heavy Higgs?, Eur. Phys. J. C 73:2354 (2013) [arXiv:1211.1955] [INSPIRE]. 
[82] Z. Kang, J. Li, T. Li, D. Liu and J. Shu, Probing the CP-even Higgs Sector via $H_{3} \rightarrow H_{2} H_{1}$ in the Natural NMSSM, arXiv:1301.0453 [INSPIRE].

[83] A. Djouadi, M. Drees, U. Ellwanger, R. Godbole, C. Hugonie et al., Benchmark scenarios for the NMSSM, JHEP 07 (2008) 002 [arXiv:0801.4321] [INSPIRE].

[84] D0 collaboration, V. Abazov et al., Search for NMSSM Higgs bosons in the $h \rightarrow a a \rightarrow \mu \mu \mu \mu, \mu \mu \tau \tau$ channels using $p \bar{p}$ collisions at $\sqrt{s}=1.96-T e V$, Phys. Rev. Lett. 103 (2009) 061801 [arXiv:0905.3381] [INSPIRE].

[85] CMS collaboration, Search for a non-standard-model Higgs boson decaying to a pair of new light bosons in four-muon final states, arXiv:1210.7619 [INSPIRE].

[86] C. Lester and D. Summers, Measuring masses of semiinvisibly decaying particles pair produced at hadron colliders, Phys. Lett. B 463 (1999) 99 [hep-ph/9906349] [INSPIRE].

[87] A. Barr, C. Lester and P. Stephens, m(T2): The Truth behind the glamour, J. Phys. G 29 (2003) 2343 [hep-ph/0304226] [INSPIRE].

[88] Heavy Flavor Averaging Group collaboration, D. Asner et al., Averages of b-hadron, c-hadron and $\tau$-lepton Properties, arXiv:1010.1589 [INSPIRE].

[89] BABAR collaboration, J. Lees et al., Evidence of $B \rightarrow \tau \nu$ decays with hadronic $B$ tags, arXiv: 1207.0698 [INSPIRE].

[90] CMS collaboration, Search for $B_{s}^{0} \rightarrow \mu^{+} \mu^{-}$and $B^{0} \rightarrow \mu^{+} \mu^{-}$decays, JHEP 04 (2012) 033 [arXiv:1203.3976] [INSPIRE].

[91] LHCB collaboration, Strong constraints on the rare decays $B_{s} \rightarrow \mu^{+} \mu^{-}$and $B^{0} \rightarrow \mu^{+} \mu^{-}$, Phys. Rev. Lett. 108 (2012) 231801 [arXiv:1203.4493] [INSPIRE].

[92] LHCB collaboration, First Evidence for the Decay $B_{s}^{0} \rightarrow \mu^{+} \mu^{-}$, Phys. Rev. Lett. 110 (2013) 021801 [arXiv:1211.2674] [INSPIRE].

[93] Heavy Flavor Averaging Group collaboration, Y. Amhis et al., Averages of B-Hadron, C-Hadron and tau-lepton properties as of early 2012, arXiv:1207.1158 [INSPIRE].

[94] Muon G-2 collaboration, G. Bennett et al., Final Report of the Muon E821 Anomalous Magnetic Moment Measurement at BNL, Phys. Rev. D 73 (2006) 072003 [hep-ex/0602035] [INSPIRE].

[95] New (G-2) collaboration, F. Gray, Measuring the muon's anomalous magnetic moment to 0.14 ppm, J. Phys. Conf. Ser. 312 (2011) 102006 [arXiv: 1009.0799] [INSPIRE].

[96] F. Jegerlehner and A. Nyffeler, The Muon g-2, Phys. Rept. 477 (2009) 1 [arXiv:0902.3360] [INSPIRE].

[97] M. Davier, A. Hoecker, B. Malaescu and Z. Zhang, Reevaluation of the Hadronic Contributions to the Muon g-2 and to alpha(MZ), Eur. Phys. J. C 71 (2011) 1515 [Erratum ibid. C 72 (2012) 1874] [arXiv:1010.4180] [INSPIRE].

[98] K. Hagiwara, R. Liao, A.D. Martin, D. Nomura and T. Teubner, $(g-2)_{\mu}$ and $\alpha\left(M_{Z}^{2}\right)$ re-evaluated using new precise data, J. Phys. G 38 (2011) 085003 [arXiv:1105.3149] [INSPIRE].

[99] WMAP collaboration, E. Komatsu et al., Seven-Year Wilkinson Microwave Anisotropy Probe (WMAP) Observations: Cosmological Interpretation, Astrophys. J. Suppl. 192 (2011) 18 [arXiv:1001.4538] [INSPIRE].

[100] XENON100 collaboration, E. Aprile et al., Dark Matter Results from 225 Live Days of XENON100 Data, Phys. Rev. Lett. 109 (2012) 181301 [arXiv:1207.5988] [INSPIRE]. 
[101] ALEPH collaboration, J. Beacham, Higgs to Four Taus at ALEPH, arXiv:1006.2491 [INSPIRE].

[102] OPAL collaboration, G. Abbiendi et al., Search for neutral Higgs boson in CP-conserving and CP-violating MSSM scenarios, Eur. Phys. J. C 37 (2004) 49 [hep-ex/0406057] [INSPIRE].

[103] OPAL collaboration, G. Abbiendi et al., Decay mode independent searches for new scalar bosons with the OPAL detector at LEP, Eur. Phys. J. C 27 (2003) 311 [hep-ex/0206022] [INSPIRE].

[104] ATLAS collaboration, A Search for Light CP-Odd Higgs Bosons Decaying to $\mu^{+} \mu^{-}$in ATLAS, ATLAS-CONF-2011-020 (2011).

[105] CMS collaboration, Search for a light pseudoscalar boson in the dimuon channel, CMS-PAS-HIG-12-004.

[106] S. Weinberg, Supersymmetry at Ordinary Energies. 1. Masses and Conservation Laws, Phys. Rev. D 26 (1982) 287 [InSPIRE].

[107] C. Aulakh and R.N. Mohapatra, Neutrino as the Supersymmetric Partner of the Majoron, Phys. Lett. B 119 (1982) 136 [INSPIRE].

[108] L.J. Hall and M. Suzuki, Explicit R-Parity Breaking in Supersymmetric Models, Nucl. Phys. B 231 (1984) 419 [InSPIRE].

[109] I.-H. Lee, Lepton Number Violation in Softly Broken Supersymmetry, Phys. Lett. B 138 (1984) 121 [INSPIRE].

[110] I.-H. Lee, Lepton Number Violation in Softly Broken Supersymmetry. 2., Nucl. Phys. B 246 (1984) 120 [InSPIRE].

[111] G.G. Ross and J. Valle, Supersymmetric Models Without R-Parity, Phys. Lett. B 151 (1985) 375 [INSPIRE].

[112] J.R. Ellis, G. Gelmini, C. Jarlskog, G.G. Ross and J. Valle, Phenomenology of Supersymmetry with Broken R-Parity, Phys. Lett. B 150 (1985) 142 [INSPIRE].

[113] P. Pandita and P.F. Paulraj, Infrared stable fixed points of Yukawa couplings in nonminimal supersymmetric standard model with R-parity violation, Phys. Lett. B 462 (1999) 294 [hep-ph/9907561] [INSPIRE].

[114] R. Kitano and K.-y. Oda, Neutrino masses in the supersymmetric standard model with right-handed neutrinos and spontaneous R-parity violation, Phys. Rev. D 61 (2000) 113001 [hep-ph/9911327] [INSPIRE].

[115] P. Pandita, Nonminimal supersymmetric standard model with baryon and lepton number violation, Phys. Rev. D 64 (2001) 056002 [hep-ph/0103005] [INSPIRE].

[116] Y. Farzan and J. Valle, R-parity violation assisted thermal leptogenesis in the seesaw mechanism, Phys. Rev. Lett. 96 (2006) 011601 [hep-ph/0509280] [INSPIRE].

[117] M. Chemtob and P. Pandita, Nonminimal supersymmetric standard model with lepton number violation, Phys. Rev. D 73 (2006) 055012 [hep-ph/0601159] [INSPIRE].

[118] B. Mukhopadhyaya and R. Srikanth, Bilarge neutrino mixing in R-parity violating supersymmetry: The Role of right-chiral neutrino superfields, Phys. Rev. D 74 (2006) 075001 [hep-ph/0605109] [INSPIRE].

[119] S. Chang and A. de Gouvêa, Neutrino alternatives for missing energy events at colliders, Phys. Rev. D 80 (2009) 015008 [arXiv:0901.4796] [INSPIRE]. 
[120] D. Lopez-Fogliani and C. Muñoz, Proposal for a new minimal supersymmetric standard model, Phys. Rev. Lett. 97 (2006) 041801 [hep-ph/0508297] [INSPIRE].

[121] N. Escudero, D.E. Lopez-Fogliani, C. Muñoz and R.R. de Austri, Analysis of the parameter space and spectrum of the mu nu SSM, JHEP 12 (2008) 099 [arXiv:0810.1507] [INSPIRE].

[122] P. Ghosh and S. Roy, Neutrino masses and mixing, lightest neutralino decays and a solution to the $\mu$ problem in supersymmetry, JHEP 04 (2009) 069 [arXiv:0812.0084] [INSPIRE].

[123] A. Bartl, M. Hirsch, A. Vicente, S. Liebler and W. Porod, LHC phenomenology of the mu nu SSM, JHEP 05 (2009) 120 [arXiv:0903.3596] [INSPIRE].

[124] P. Bandyopadhyay, P. Ghosh and S. Roy, Unusual Higgs boson signal in R-parity violating nonminimal supersymmetric models at the LHC, Phys. Rev. D 84 (2011) 115022 [arXiv: 1012.5762] [INSPIRE].

[125] P. Ghosh, D.E. Lopez-Fogliani, V.A. Mitsou, C. Muñoz and R.R. de Austri, Displaced multileptons at the $\mathrm{LHC}$ - probing a 125 GeV new boson in $\mu \nu S S M$, arXiv:1211.3177 [INSPIRE].

[126] J. Fidalgo, D.E. Lopez-Fogliani, C. Muñoz and R.R. de Austri, The Higgs sector of the $\mu \nu S S M$ and collider physics, JHEP 10 (2011) 020 [arXiv:1107.4614] [INSPIRE].

[127] B. Mukhopadhyaya, S. Roy and F. Vissani, Correlation between neutrino oscillations and collider signals of supersymmetry in an R-parity violating model, Phys. Lett. B 443 (1998) 191 [hep-ph/9808265] [INSPIRE].

[128] E.J. Chun and J.S. Lee, Implication of Super-Kamiokande data on R-parity violation, Phys. Rev. D 60 (1999) 075006 [hep-ph/9811201] [INSPIRE].

[129] S. Choi, E.J. Chun, S.K. Kang and J.S. Lee, Neutrino oscillations and R-parity violating collider signals, Phys. Rev. D 60 (1999) 075002 [hep-ph/9903465] [INSPIRE].

[130] J. Romao, M. Diaz, M. Hirsch, W. Porod and J. Valle, A Supersymmetric solution to the solar and atmospheric neutrino problems, Phys. Rev. D 61 (2000) 071703 [hep-ph/9907499] [INSPIRE].

[131] W. Porod, M. Hirsch, J. Romao and J. Valle, Testing neutrino mixing at future collider experiments, Phys. Rev. D 63 (2001) 115004 [hep-ph/0011248] [INSPIRE].

[132] M. Hirsch, A. Vicente and W. Porod, Spontaneous R-parity violation: Lightest neutralino decays and neutrino mixing angles at future colliders, Phys. Rev. D 77 (2008) 075005 [arXiv:0802.2896] [INSPIRE].

[133] D. G. Cerdeño, P. Ghosh, C. B. Park and M. Peiró, in preparation.

[134] U. Ellwanger, J.F. Gunion and C. Hugonie, NMHDECAY: A Fortran code for the Higgs masses, couplings and decay widths in the NMSSM, JHEP 02 (2005) 066 [hep-ph/0406215] [INSPIRE].

[135] U. Ellwanger and C. Hugonie, NMHDECAY 2.0: An Updated program for sparticle masses, Higgs masses, couplings and decay widths in the NMSSM, Comput. Phys. Commun. 175 (2006) 290 [hep-ph/0508022] [INSPIRE].

[136] U. Ellwanger and C. Hugonie, NMSPEC: A Fortran code for the sparticle and Higgs masses in the NMSSM with GUT scale boundary conditions, Comput. Phys. Commun. 177 (2007) 399 [hep-ph/0612134] [INSPIRE].

[137] A. Djouadi, M. Spira and P. Zerwas, Production of Higgs bosons in proton colliders: QCD corrections, Phys. Lett. B 264 (1991) 440 [InSPIRE]. 
[138] M. Spira, A. Djouadi, D. Graudenz and P. Zerwas, Higgs boson production at the LHC, Nucl. Phys. B 453 (1995) 17 [hep-ph/9504378] [INSPIRE].

[139] A. Djouadi, M. Spira and P. Zerwas, QCD corrections to hadronic Higgs decays, Z. Phys. C 70 (1996) 427 [hep-ph/9511344] [INSPIRE].

[140] A. Djouadi, J. Kalinowski and M. Spira, HDECAY: A Program for Higgs boson decays in the standard model and its supersymmetric extension, Comput. Phys. Commun. 108 (1998) 56 [hep-ph/9704448] [INSPIRE].

[141] M. Muhlleitner, A. Djouadi and Y. Mambrini, SDECAY: A Fortran code for the decays of the supersymmetric particles in the MSSM, Comput. Phys. Commun. 168 (2005) 46 [hep-ph/0311167] [INSPIRE].

[142] M. Muhlleitner, SDECAY: A Fortran code for SUSY particle decays in the MSSM, Acta Phys. Polon. B 35 (2004) 2753 [hep-ph/0409200] [InSPIRE].

[143] D. Das, U. Ellwanger and A.M. Teixeira, NMSDECAY: A Fortran Code for Supersymmetric Particle Decays in the Next-to-Minimal Supersymmetric Standard Model, Comput. Phys. Commun. 183 (2012) 774 [arXiv:1106.5633] [INSPIRE].

[144] G. Bélanger, F. Boudjema, A. Pukhov and A. Semenov, MicrOMEGAs 2.0: A Program to calculate the relic density of dark matter in a generic model,

Comput. Phys. Commun. 176 (2007) 367 [hep-ph/0607059] [INSPIRE].

[145] G. Bélanger, F. Boudjema, A. Pukhov and A. Semenov, Dark matter direct detection rate in a generic model with MicrOMEGAs 2.2, Comput. Phys. Commun. 180 (2009) 747 [arXiv:0803.2360] [INSPIRE].

[146] C. Bobeth, A.J. Buras, F. Krüger and J. Urban, $Q C D$ corrections to $\bar{B} \rightarrow X_{d, s} \nu \bar{\nu}$, $\bar{B}_{d, s} \rightarrow \ell^{+} \ell^{-}, K \rightarrow \pi \nu \bar{\nu}$ and $K_{L} \rightarrow \mu^{+} \mu^{-}$in the MSSM, Nucl. Phys. B 630 (2002) 87 [hep-ph/0112305] [INSPIRE].

[147] G. Hiller, B physics signals of the lightest CP odd Higgs in the NMSSM at large tan beta, Phys. Rev. D 70 (2004) 034018 [hep-ph/0404220] [INSPIRE].

[148] F. Domingo and U. Ellwanger, Updated Constraints from B Physics on the MSSM and the NMSSM, JHEP 12 (2007) 090 [arXiv:0710.3714] [INSPIRE].

[149] R.N. Hodgkinson and A. Pilaftsis, Supersymmetric Higgs Singlet Effects on B-Meson FCNC Observables at Large $\tan \beta$, Phys. Rev. D 78 (2008) 075004 [arXiv:0807.4167] [INSPIRE].

[150] M. Misiak, H. Asatrian, K. Bieri, M. Czakon, A. Czarnecki et al., Estimate of $B(\bar{B} \rightarrow X(s) \gamma)$ at $O\left(\alpha_{s}^{2}\right)$, Phys. Rev. Lett. 98 (2007) 022002 [hep-ph/0609232] [INSPIRE].

[151] M. Misiak and M. Steinhauser, NNLO QCD corrections to the $\bar{B} \rightarrow X_{s} \gamma$ matrix elements using interpolation in $m_{c}$, Nucl. Phys. B 764 (2007) 62 [hep-ph/0609241] [INSPIRE].

[152] ATLAS collaboration, Multi-channel search for squarks and gluinos in $\sqrt{s}=7 \mathrm{TeV} p p$ collisions with the ATLAS detector, Eur. Phys. J. C 73 (2013) 2362 [arXiv:1212.6149] [INSPIRE].

[153] CMS collaboration, Search for new physics in the multijet and missing transverse momentum final state in proton-proton collisions at $\sqrt{s}=7 \mathrm{TeV}$,

Phys. Rev. Lett. 109 (2012) 171803 [arXiv:1207.1898] [INSPIRE].

[154] ATLAS collaboration, An update of combined measurements of the new Higgs-like boson with high mass resolution channels, ATLAS-CONF-2012-170 (2012). 
[155] D.M. Lindemann, $B+\rightarrow \tau \nu$ and $B \rightarrow K(*) \nu \bar{\nu}$ at BaBar and SuperB, arXiv:1212.5578 [INSPIRE].

[156] M. Bahr, S. Gieseke, M. Gigg, D. Grellscheid, K. Hamilton et al., HERWIG++ Physics and Manual, Eur. Phys. J. C 58 (2008) 639 [arXiv:0803.0883] [INSPIRE].

[157] K. Arnold, L. d'Errico, S. Gieseke, D. Grellscheid, K. Hamilton et al., HERWIG++ 2.6 Release Note, arXiv:1205.4902 [INSPIRE].

[158] J. Pumplin, D. Stump, J. Huston, H. Lai, P.M. Nadolsky et al., New generation of parton distributions with uncertainties from global QCD analysis, JHEP 07 (2002) 012 [hep-ph/0201195] [INSPIRE].

[159] M. Spira, HIGLU: A program for the calculation of the total Higgs production cross-section at hadron colliders via gluon fusion including QCD corrections, hep-ph/9510347 [INSPIRE].

[160] S. Ovyn, X. Rouby and V. Lemaitre, DELPHES, a framework for fast simulation of a generic collider experiment, arXiv:0903.2225 [INSPIRE].

[161] M. Cacciari, G.P. Salam and G. Soyez, The Anti-k(t) jet clustering algorithm, JHEP 04 (2008) 063 [arXiv:0802.1189] [InSPIRE].

[162] T. Sjöstrand, S. Mrenna and P.Z. Skands, PYTHIA 6.4 Physics and Manual, JHEP 05 (2006) 026 [hep-ph/0603175] [INSPIRE].

[163] P. Baringer, K. Kong, M. McCaskey and D. Noonan, Revisiting Combinatorial Ambiguities at Hadron Colliders with $M_{T 2}$, JHEP 10 (2011) 101 [arXiv:1109.1563] [INSPIRE].

[164] K. Choi, D. Guadagnoli and C.B. Park, Reducing combinatorial uncertainties: A new technique based on MT2 variables, JHEP 11 (2011) 117 [arXiv:1109.2201] [INSPIRE].

[165] V.D. Barger, T. Han and J. Ohnemus, Heavy leptons at hadron supercolliders, Phys. Rev. D 37 (1988) 1174 [INSPIRE].

[166] W.S. Cho, K. Choi, Y.G. Kim and C.B. Park, M(T2)-assisted on-shell reconstruction of missing momenta and its application to spin measurement at the LHC, Phys. Rev. D 79 (2009) 031701 [arXiv: 0810.4853] [InSPIRE].

[167] K. Choi, S. Choi, J.S. Lee and C.B. Park, Reconstructing the Higgs boson in dileptonic W decays at hadron collider, Phys. Rev. D 80 (2009) 073010 [arXiv: 0908.0079] [INSPIRE].

[168] K. Choi, J.S. Lee and C.B. Park, Measuring the Higgs boson mass with transverse mass variables, Phys. Rev. D 82 (2010) 113017 [arXiv: 1008.2690] [INSPIRE].

[169] C.B. Park, Reconstructing the heavy resonance at hadron colliders, Phys. Rev. D 84 (2011) 096001 [arXiv:1106.6087] [INSPIRE]. 